ARTICLE

\title{
The ribosome-associated complex RAC serves in a relay that directs nascent chains to Ssb
}

Ying Zhang ${ }^{1,3}$, Genís Valentín Gesé (i) ${ }^{2,3}$, Charlotte Conz ${ }^{1}$, Karine Lapouge (1) ${ }^{2}$, Jürgen Kopp², Tina Wölfle , Sabine Rospert ${ }^{1 凶} \&$ Irmgard Sinning (1) ${ }^{2 凶}$

The conserved ribosome-associated complex (RAC) consisting of Zuo1 (Hsp40) and Ssz1 (non-canonical Hsp70) acts together with the ribosome-bound Hsp70 chaperone Ssb in de novo protein folding at the ribosomal tunnel exit. Current models suggest that the function of Ssz1 is confined to the support of Zuo1, however, it is not known whether RAC by itself serves as a chaperone for nascent chains. Here we show that, via its rudimentary substrate binding domain (SBD), Ssz1 directly binds to emerging nascent chains prior to Ssb. Structural and biochemical analyses identify a conserved LP-motif at the Zuo1 N-terminus forming a polyproline-II helix, which binds to the Ssz1-SBD as a pseudo-substrate. The LP-motif competes with nascent chain binding to the Ssz1-SBD and modulates nascent chain transfer. The combined data indicate that Ssz1 is an active chaperone optimized for transient, low-affinity substrate binding, which ensures the flux of nascent chains through RAC/Ssb.

\footnotetext{
${ }^{1}$ Institute of Biochemistry and Molecular Biology, ZBMZ, Faculty of Medicine, University of Freiburg, D-79104 Freiburg, Germany; BIOSS Centre for Biological Signaling Studies, University of Freiburg, D-79104 Freiburg, Germany. ${ }^{2}$ Heidelberg University Biochemistry Center (BZH), INF 328, D-69120 Heidelberg, Germany.

${ }^{3}$ These authors contributed equally: Ying Zhang, Genís Valentín Gesé. ${ }^{凶}$ email: sabine.rospert@biochemie.unifreiburg.de; irmi.sinning@bzh.uni-heidelberg.de
} 
R ibosome-associated chaperones play a fundamental role in de novo protein folding in all kingdoms of life. They encounter nascent polypeptides when they emerge from the ribosomal exit tunnel and act before cytosolic chaperones become involved in folding as translation proceeds ${ }^{1,2}$. Eukaryotic cells possess a specialized ribosome-associated complex (RAC), which in yeast is a stable heterodimer formed by the Hsp70 homolog Ssz1 and the J-domain protein $\mathrm{Zuol}^{3,4}$ (Fig. 1a). RAC is the J-domain partner of the ribosome-associated Hsp70 homolog Ssb (encoded by two nearly identical isoforms SSB1 and SSB2). Ssb cotranslationally interacts with a variety of nascent chains when they emerge at the ribosomal tunnel exit ${ }^{3,5-7}$. Binding of Ssb to nascent chains depends on an intact Zuol J-domain and Ssz1 ${ }^{8}$. Consistently, both RAC subunits are required to efficiently stimulate ATP hydrolysis by $\mathrm{Ssb}^{9}$.

$\mathrm{Ssb}$ is a canonical Hsp70 protein comprising an N-terminal nucleotide binding domain (NBD), an interdomain linker, and a C-terminal substrate binding domain (SBD), composed of SBD $\beta$ and the SBDa lid domain ${ }^{3,4,10}$. Ssb contacts ribosomal proteins and rRNA segments, which localize around the tunnel exit ${ }^{4,10}$ in proximity to $\mathrm{RAC}^{11,12}$. Zuol possesses a complex domain structure with a long a-helical middle domain (MD), which separates two major functional domains. The N-terminal part comprises an N-terminal domain $(\mathrm{N})$, the J-domain $(\mathrm{J})$ and the zuotin homology domain (ZHD). Zuo1 contacts the $60 \mathrm{~S}$ subunit via the ZHD close to Rpl31 (eL31) and H24 of the $25 \mathrm{~S}$ rRNA at the tunnel exit ${ }^{4,11-13}$. The Zuo1 C-terminal domain consists of a 4-helix bundle (HD) ${ }^{14,15}$, which contacts the $40 \mathrm{~S}$ subunit at the tip of h44 ES12 4,12 . Sszl significantly differs from canonical Hsp70 homologs. The Ssz1-NBD binds ATP, but does not hydrolyze ATP 9,14 . Our recent structure of the RAC core (consisting of Ssz1 and Zuo1N residues 19 to 60) shows that the Ssz1 interdomain linker, which is critical for allosteric regulation of canonical Hsp70s ${ }^{16}$ has a unique structure and is detached from the $\mathrm{NBD}^{17}$. Moreover, Ssz1 lacks the C-terminal SBDa lid domain, but contains a complete $\operatorname{SBD} \beta$ complemented by ZuolN ${ }^{17}$. Thus, from all we know, Ssz1 cannot undergo the canonical Hsp70 cycle of structural rearrangements required for substrate binding and release ${ }^{18}$. Besides, in vitro crosslinking experiments, which readily reveal the interaction of Ssb with nascent chains, up to now did not reveal an interaction of Ssz1 with nascent chains ${ }^{8}$. Based on these observations the current model is that Ssz1 does not act as a typical Hsp70 chaperone. Instead, it may primarily play a structural role, supporting Zuol function as the J-domain partner of $\mathrm{Ssb}^{9,19}$.

Here we investigate whether the RAC subunits interact with nascent chains. Our results indicate that both RAC subunits contact nascent chains and form a relay that transfers them from Ssz1 to Ssb. Structural analysis reveals that the Zuo1 N-terminus contains a conserved motif (LP-motif) which binds to the Ssz1$\mathrm{SBD} \beta$ in the same way as canonical Hsp70s bind their substrates. This motif competes with nascent chain binding, and modulates the interaction of the nascent chain with Zuol and Ssz1. Overall, we show that Sszl is an active chaperone with specific deviations from the canonical Hsp70 mechanism.

\section{Results}

Nascent chains contact Zuol and Ssz1 prior to Ssb. As the structure of the RAC core shows the presence of a complete $\operatorname{SBD} \beta^{17}$, RAC should be able to bind substrates with low affinity besides its function as J-domain partner of Ssb. To test for the possibility, we generated ribosome nascent chain complexes (RNCs) and analyzed nascent chain contacts via a crosslinking approach using Saccharomyces cerevisiae $(\mathrm{Sc})$ as a model system (Fig. 1b) ${ }^{20}$. As a substrate we employed the soluble, cytosolic enzyme phosphoglycerate kinase (Pgk1), which is a well characterized nascent chain ${ }^{20,21}$. As expected, Ssb formed a weak crosslink to 50 residues nascent Pgk1 (Pgk1-50), while Ssb formed more efficient crosslinks with 100- or 150 residues nascent Pgk1, respectively ${ }^{5,6}$. Consistent with previous crosslinking analysis of nascent prepro $\alpha$-factor ${ }^{8}$, crosslinks between Zuol or Ssz1 and nascent Pgk1-50, or longer, were below the detection limit (Supplementary Fig. 1a).

We next tested the interaction of Ssb and the RAC subunits with nascent Pgk1-40 and Pgk1-45, respectively. Ssb did not form crosslink products with Pgk1-40 or Pgk1-45 (Fig. 1c). This is consistent with previous data indicating that the interaction with Ssb requires a minimal nascent chain length of about 50 residues $^{5-7}$. However, Zuol and Sszl formed crosslinks with these short nascent chains (Fig. 1c). Zuol was most efficiently crosslinked to Pgk1-40, while Ssz1 was most efficiently crosslinked to Pgk1-45 (Fig. 1c). These crosslinks disappeared at the expense of the Ssb crosslink when the nascent chain reached a length of 50 residues (Fig. 1c). The data suggested that the nascent chain was transferred via a chain of interactions from Zuo1, to Ssz1, and finally to Ssb.

To further investigate the handover process, we tested whether RAC was in contact with longer nascent chains when Ssb was absent, i.e. when nascent chains were generated in a translation extract derived from $\Delta s s b 1 \Delta s s b 2$ cells (Fig. 1d, Supplementary Fig. 1b). Indeed, when Ssb was absent, Zuo1 and Ssz1 formed efficient crosslinks to nascent chains of 50-150 residues (Fig. 1d $\Delta s s b$ and Supplementary Fig. 1b). Seemingly, Ssb prevented the interaction of Zuol and Ssz1 with long nascent chains, which is consistent with a direct and efficient nascent chain transfer from RAC to Ssb. To further corroborate this model, we used an Ssb mutant, which carries a deletion of its C-terminal 23 residues (Ssb- $\Delta$ C23), which does not stably interact with ribosomes (Supplementary Fig. 1c and 1d) ${ }^{10,22}$. When nascent chains were generated in a translation extract derived from Ssb- $\Delta$ C23 cells, no crosslink between Ssb- $\Delta$ C23 and Pgk1-100 was detected, but instead nascent Pgk1-100 remained in close contact with the RAC subunits (Fig. 1d). Thus, efficient crosslinking between the nascent chain and Ssb was dependent on the positioning of Ssb at the exit of the ribosomal tunnel. If Ssb was not bound to the ribosomal tunnel exit region, the nascent chain maintained its contacts with the RAC subunits.

We next asked if both subunits of RAC were required for the handover to ribosome-bound Ssb. To that end, we analyzed the interaction of Ssb1 with nascent Pgk1-100 (i) in the absence of both RAC subunits; (ii) in the presence of the N-terminally truncated Zuo1- $\Delta$ N49 mutant, which does not stably interact with Ssz1 leading to a situation in which Zuol is ribosome-bound, but Ssz1 is not ${ }^{17}$ (Supplementary Fig. 1e, Zuol- $\Delta$ N49); and (iii) in the presence of Zuo1, but absence of Ssz1 (Fig. 1e). Only in the presence of wild type RAC nascent Pgk1-100 formed an efficient crosslink to Ssb (Fig. 1e, XL-Ssb). When Ssz1 was absent or was not in a stable complex with Zuol the nascent chain remained in contact with Zuo1, even though Ssb was associated with ribosomes (Fig. 1e, XL-Zuo1; Supplementary Fig. 1f). The findings strongly suggested that Ssz1 was required for the handover from Zuol to ribosome-bound Ssb. This was further corroborated when nascent Pgk1-100 was generated in a translation extract derived from a $\Delta s s b 1 \Delta s s b 2$ strain and purified Ssb1 was added prior to the translation reaction (Fig. 1f; Supplementary Fig. 1g,h). Consistent with the data shown above both RAC subunits formed crosslinks with the nascent chain in the absence (Fig. 1f, $\Delta s s b$ extract), but not in the presence of Ssb (Fig. 1f, wt). When purified Ssb (Supplementary Fig. 1g) was added to the $\Delta s s b 1 \Delta s s b 2$ extract, crosslinking to the RAC subunits was strongly reduced and crosslinking of Ssb to nascent Pgk1-100 was restored (Fig. 1f, 
Supplementary Fig. 1h). Taken together, these data reveal three novel functions of RAC. First, both RAC subunits are in close contact with nascent chains when these emerge at the tunnel exit. Second, the RAC subunits remain in contact with the nascent chain for a prolonged period of time, when Ssb is absent or is not bound to the ribosome. Third, the presence and proper positioning of Sszl as well as Ssb at the tunnel exit is required for the transfer of the growing nascent chain from Zuo1 to Ssb.

Zuo1N extends the Ssz1-SBD $\beta$. Having established that Ssz1 and Zuol both interact with nascent chains, we aimed to understand a

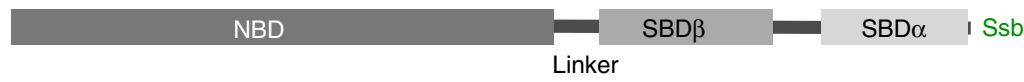

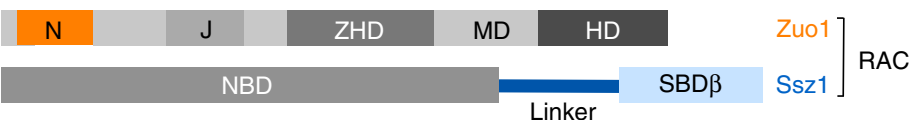

C Nascent Pgk1-

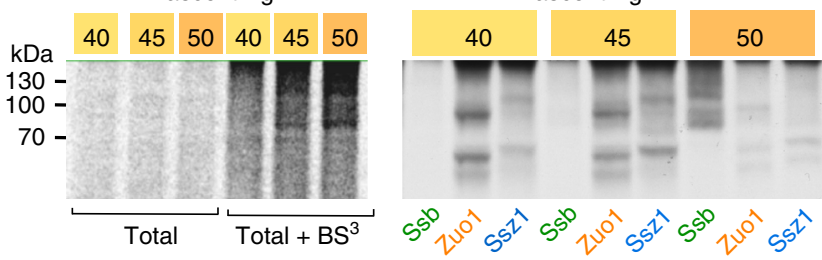

d

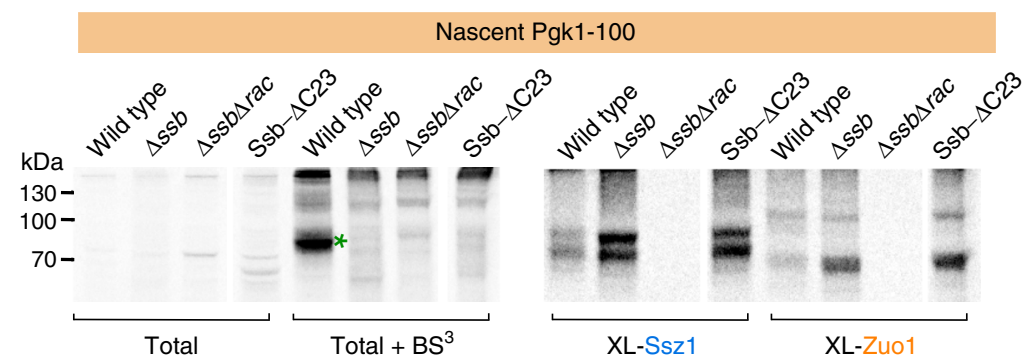

f

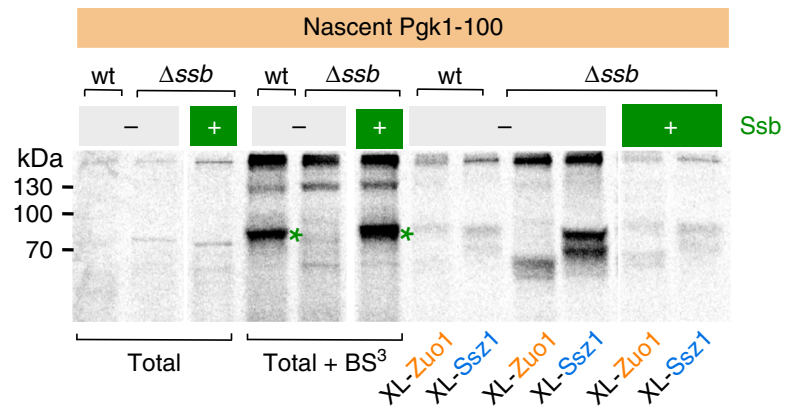

b

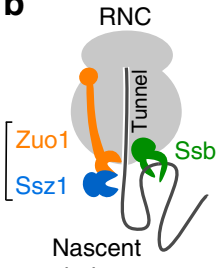

chain

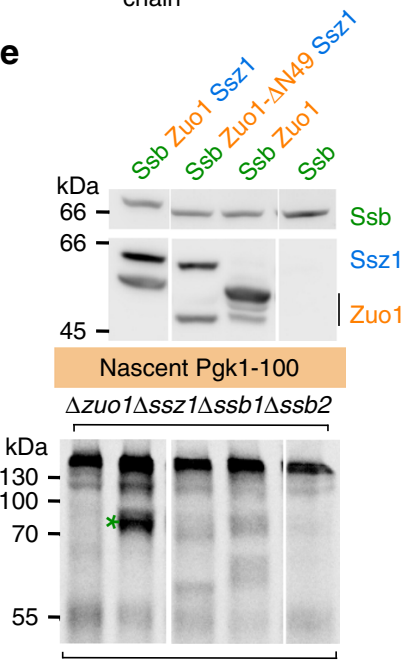

Total $+\mathrm{BS}^{3}$
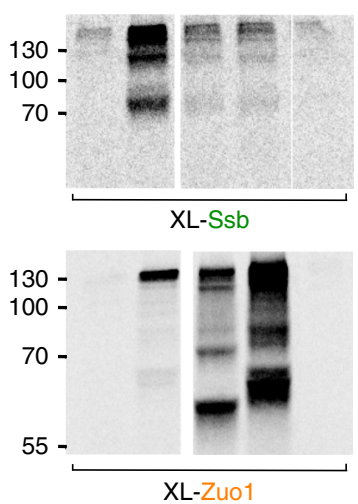

Fig. 1 Zuo1 and Ssz1 contact nascent chains prior to Ssb. a Domain structure of yeast Ssb, Zuo1, and Ssz1. Nucleotide binding domain (NBD), linker, substrate binding domain $\beta(S B D \beta)$, substrate binding domain $\alpha(S B D \alpha), N$-terminal domain $(N)$, J-domain (J), Zuo1 homology domain (ZHD), middle domain (MD), and four-helix bundle (HD). b Experimental set up of crosslinking experiments. Isolated RNCs carrying [ $\left.{ }^{35} \mathrm{~S}\right]$-labelled nascent chains are crosslinked to adjacent proteins using the homobifunctional amino-reactive crosslinker BS ${ }^{3}$, spacer length $11.4 \AA$. Crosslink products between nascent chains and Ssb, Ssz1, or Zuo1 are then identified via immunoprecipitation under denaturing conditions using antibodies directed against Ssb, Ssz1, or Zuo1. For details see Methods. c Contacts of Ssb, Zuo1, and Ssz1 with short nascent chains. RNCs carrying Pgk1-40 (40 residues), -45 (45 residues), or -50 ( 50 residues) were generated in a wild type translation extract as described in (b). Crosslink products (Pgk1-XL) between Ssb and nascent Pgk1 (Ssb); Zuo1 and nascent Pgk1 (Zuo1); and Ssz1 and nascent Pgk1 (Ssz1). d Zuo1 and Ssz1 interact with nascent Pgk1-100 (100 residues) in the absence of ribosome-bound Ssb. The experiment was performed as described in c using RNCs from wild type, $\Delta s s b 1 \Delta s s b 2(\Delta s s b), \Delta s s b 1 \Delta s s b 2 \Delta z u 01 \Delta s s z 1$ ( $\Delta s s b \Delta r a c)$, or Ssb1- $\Delta$ C23 translation extract. The green asterisk indicates the crosslink between Ssb and Pgk1-100 in the total (d-f). e Transfer of the nascent chain from Zuo1 to Ssb requires Ssz1. The experiment was performed as in (c) using RNCs from $\Delta z u 01 \Delta s s z 1 \Delta s s b 1 \Delta s s b 2$ translation extract. Ribosome-free extracts (upper panel) obtained from wild type (Ssb, Zuo1, Ssz1), $\Delta$ zuo1 expressing Zuo1- $\Delta$ N49 (Ssb, Zuo1- $\Delta$ N49, Ssz1), $\Delta$ ssz1 (Ssb, Zuo1), $\Delta$ zuo1 $\Delta s s z 1$ (Ssb) were added after completion of translation reactions, prior to crosslinking. Rebinding of Ssb to RNCs was analyzed as described in Supplementary Fig. If. f Ssb prevents crosslinking of Zuo1 and Ssz1 to long nascent chains. The experiment was performed as in (c) using RNCs from wild type (wt) or $\Delta s s b 1 \Delta s s b 2$ ( $\Delta s s b$ ) translation extract. Purified Ssb1 (Supplementary Fig. 1g,h) was added prior to the translation reaction as indicated (+ Ssb). Source data for (c-e) are provided as a Source Data file. 
a

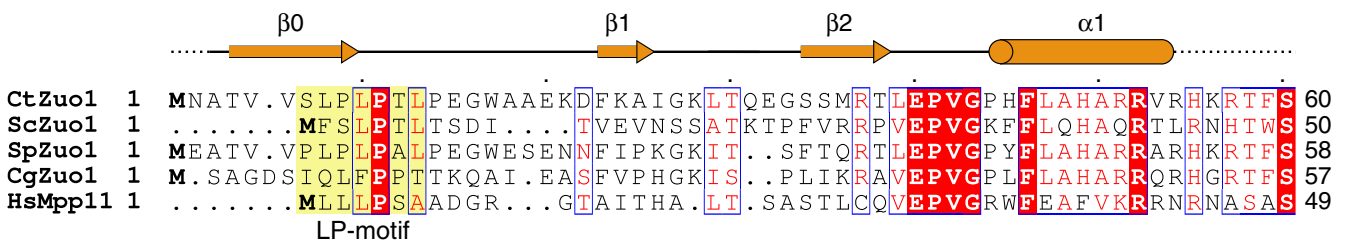

b

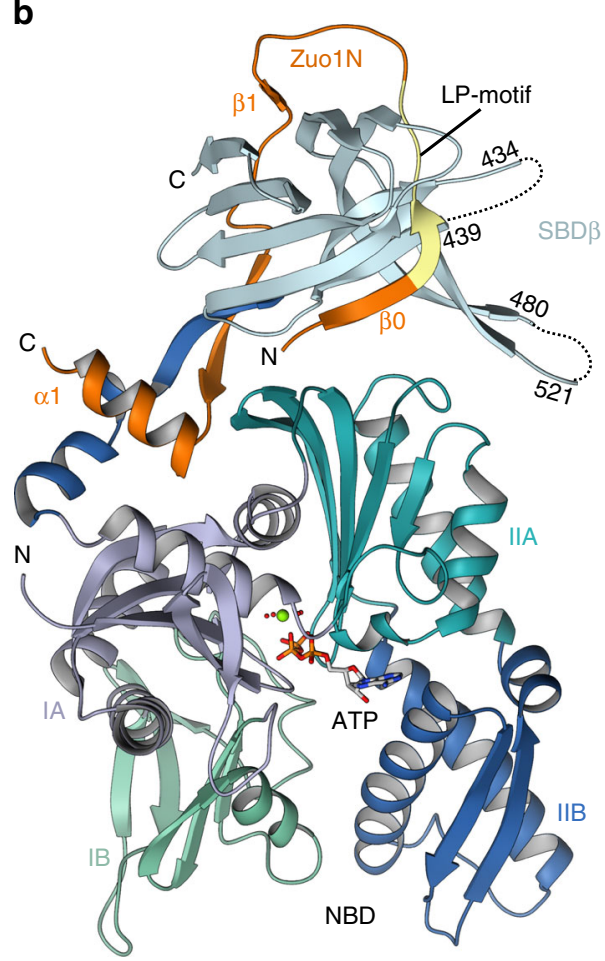

e

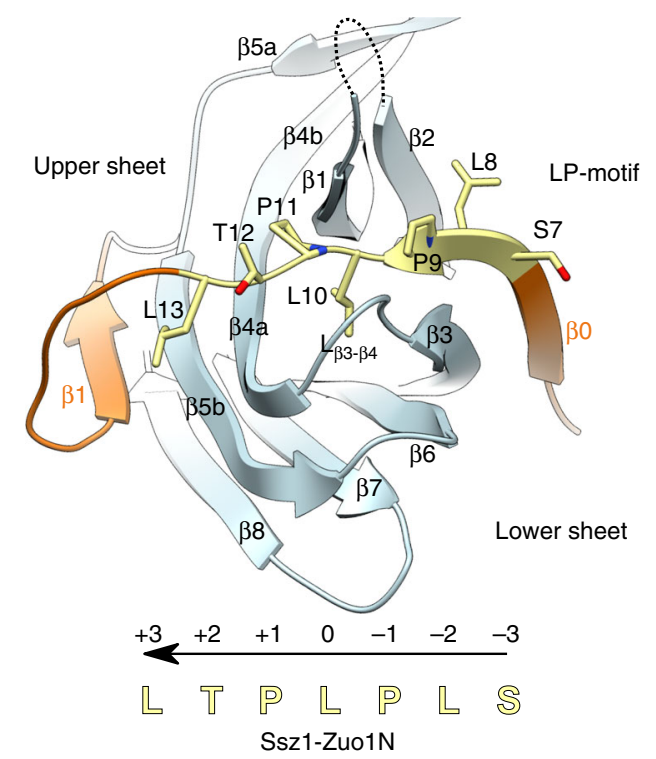

how RAC could accommodate a nascent chain. Previous structural data of the RAC core from Chaetomium thermophilum $(\mathrm{Ct})$, consisting of full length CtSsz1 and CtZuo1N (residues 19-60), showed that it contains a complete Ssz1-SBD $\beta$-sandwich domain, which should allow for low-affinity substrate binding ${ }^{17}$. Complementation of the Ssz1-SBD $\beta$ by ZuolN in trans creates a mixed RAC-SBD with a topology seen in canonical Hsp70s in the

d
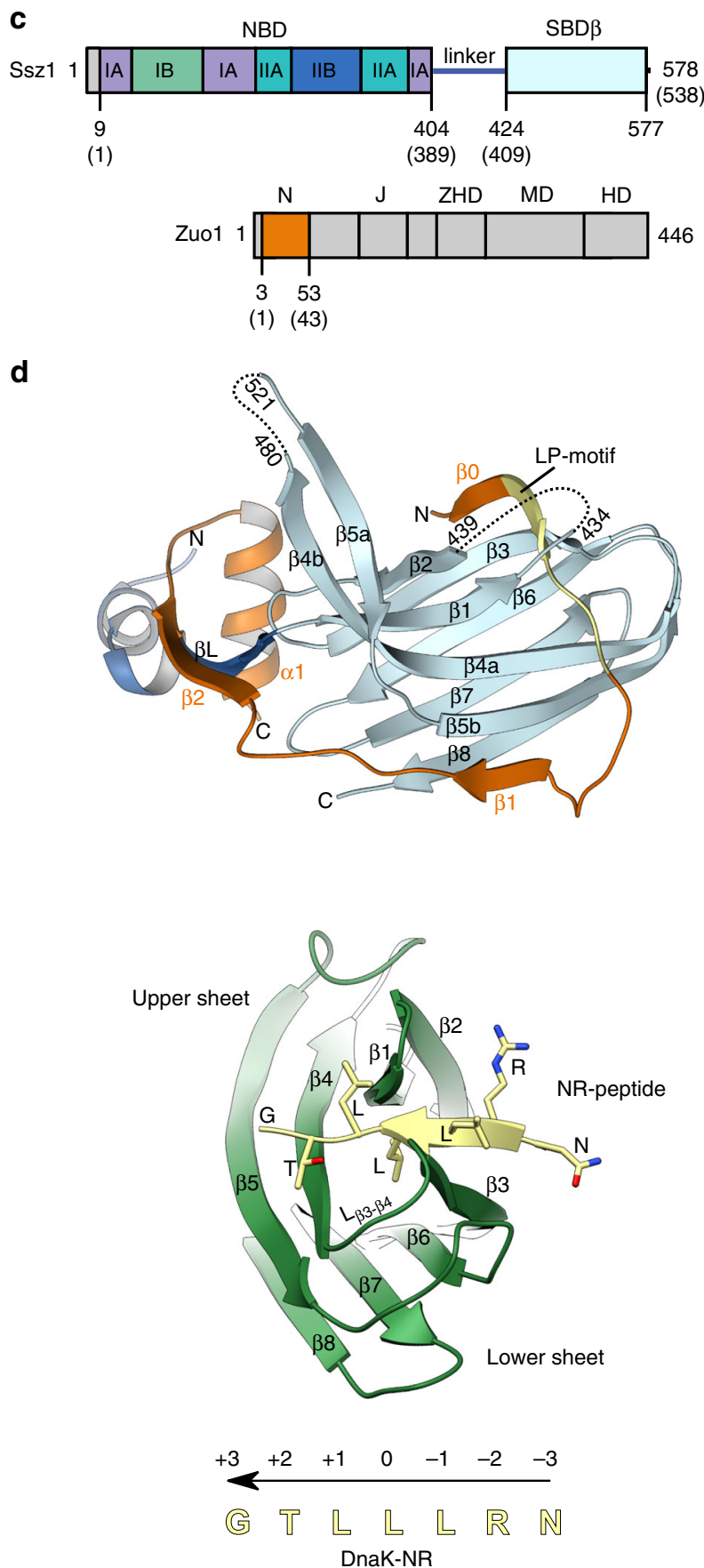

closed (ADP-bound) state. However, attempts to bind typical Hsp70 substrate peptides to RAC in vitro failed ${ }^{17}$.

We now determined the structure of an N-terminally extended RAC core comprising CtSsz1 with the complete CtZuo1N (residues 1-60 of CtZuol; Fig. 2a,b) at $2.5 \AA$ resolution. The structure has been solved by molecular replacement using the previous RAC core as a search model (Fig. $2 \mathrm{a}-\mathrm{d}$ and Table 1). 
Fig. 2 The structure of the Ssz1-Zuo1N complex reveals binding of a pseudo-substrate. a Multiple sequence alignment of Zuo1N. The secondary structure derived from the crystal structure is depicted above. Ct, Chaetomium thermophilum; Sc, Saccharomyces cerevisiae; Sp, Schizosaccharomyces pombe; $\mathrm{Cg}$, Chaetomium globosum; Hs, Homo sapiens. The alignment is color coded by conservation: strictly conserved residues are in a red box, similar residues are in red font. The LP-motif is highlighted in yellow. b Crystal structure of CtSsz1-Zuo1N in ribbon representation. The Ssz1-NBD, Ssz1-SBD $\beta$ and Zuo1N are colored as in (c). The LP-motif is highlighted in yellow. Disordered residues are indicated as dotted lines. The ATP, Mg ${ }^{2+}$ and coordinated waters are shown in stick and sphere representation. c Domain architecture of Ssz1 and Zuo1. Domains present in the crystal structure are indicated by residue numbers for Ct (Sc numbers are in parentheses). Domain designation is as in Fig. 1a. d Zoomed-in view on the interaction of Ssz1-SBD $\beta$ with Zuo1N, showing how Zuo1N extends the SBD $\beta$. Coloring is as in (c). e Comparison of substrate binding to Ssz1-SBD $\beta$ (left) and DnaK (right) (23; PDB 1DKZ). The $\mathrm{SBD} \alpha$ of DnaK is not shown. Side chains of the LP-motif and NR-peptide are shown as stick representations. Sequences and direction (N-to C-terminus) of the LP-motif and NR-peptide are shown below the structural representations. Numbers indicate the position of each residue with respect to the leucine that occupies the center of the substrate binding pocket (position 0). Coloring of Ssz1 and Zuo1N is as in (c). DnaK is shown in green and the NR-peptide in yellow.

\begin{tabular}{|c|c|}
\hline & Ssz1-Zuo1N \\
\hline \multicolumn{2}{|l|}{ Data collection } \\
\hline Space group & $P 1211$ \\
\hline \multicolumn{2}{|l|}{ Cell dimensions } \\
\hline$a, b, c(\AA)$ & $52.0,258.5,53.0$ \\
\hline$\alpha, \beta, \gamma\left(^{\circ}\right)$ & $90.0,100.1,90.0$ \\
\hline Resolution $(\AA)$ & $48.40-2.5(2.59-2.5)$ \\
\hline Rmerge & $13.4(70.6)$ \\
\hline $\mathrm{I} / \sigma(\mathrm{I})$ & $9.6(2.2)$ \\
\hline $\mathrm{CC}_{1 / 2}(\%)$ & $99.2(65.8)$ \\
\hline Completeness (\%) & $98.9(96.8)$ \\
\hline Redundancy & $6.0(5.5)$ \\
\hline \multicolumn{2}{|l|}{ Refinement } \\
\hline Resolution $(\AA)$ & $48.40-2.5(2.59-2.5)$ \\
\hline No. reflections & $46791(4582)$ \\
\hline$R_{\text {work }} / R_{\text {free }}(\%)$ & $18.89 / 22.34$ \\
\hline \multicolumn{2}{|l|}{ No. atoms } \\
\hline Protein & 8732 \\
\hline lons $\left(\mathrm{Mg}^{2+}\right)$ & 2 \\
\hline Ligands (ATP) & 62 \\
\hline Water & 91 \\
\hline \multicolumn{2}{|l|}{ B factors $\left(\AA^{2}\right)$} \\
\hline Protein & 53.71 \\
\hline Ion $\left(\mathrm{Mg}^{2+}\right)$ & 34.3 \\
\hline Ligand (ATP) & 34.56 \\
\hline Water & 36.3 \\
\hline \multicolumn{2}{|l|}{ R.m.s. deviations } \\
\hline Bond lengths $(\AA)$ & 0.004 \\
\hline Bond angles $\left({ }^{\circ}\right)$ & 0.98 \\
\hline
\end{tabular}

Each structure was determined from one crystal.

Values in parentheses are for the highest-resolution shell.

Overall, the structure of Ssz1-Zuo1N ${ }^{1-60}$ superimposes well with the previous structure ${ }^{17}$ (Supplementary Fig. 2a,b). The Ssz1NBD adopts the typical actin-like fold of Hsp70s, which is divided into two lobes (I and II), with ATP and $\mathrm{Mg}^{2+}$ bound between them (Fig. 2b). The domain arrangement is as before, with the Ssz1 linker detached from the Ssz1-NBD creating a loophole for ZuolN and with the conserved Zuol EPVG motif contacting the Ssz1-NBD at the linker binding site (Fig. 2b, Supplementary Fig. 2b).

However, the structure of the Ssz1-Zuo1N ${ }^{1-60}$ complex reveals a number of novel, functionally important details. First, Ssz1-SBD $\beta 8$ is now ordered and results in an Ssz1 $\beta$-sandwich composed of a 4 -stranded upper $(\beta 2, \beta 1, \beta 4 \mathrm{a}, \beta 5 \mathrm{~b})$, and a 4 -stranded lower sheet $(\beta 3, \beta 6, \beta 7, \beta 8)$ (Fig. $2 \mathrm{~d}$ and Supplementary Fig. $2 \mathrm{c}, \mathrm{d})$ as characteristic for canonical Hsp70-SBD $\beta$ s in the closed state ${ }^{23,24}$. In contrast to the previous structure (Ssz1-Zuo1N $\left.{ }^{19-60}\right)$ in which Ssz1 $\beta 8$ was disordered and its position taken by Zuo1N $\beta 1^{17}$,
Ssz1 $\beta 8$ is now structured and aligns with $\beta 7$ of the lower sheet. Second, the N-terminal extension of Zuo1N contributes two additional $\beta$ strands $(\beta 0, \beta 1)$ to $\operatorname{Ssz} 1-\operatorname{SBD} \beta$ resulting in a $\beta$ sandwich with two mixed, 5-stranded $\beta$ sheets. Zuo1N $\beta 0$ complements the lower and $\beta 1$ now the upper sheet of Ssz1SBD $\beta$, aligning with Ssz1-SBD $\beta 3$ and $\beta 5 b$, respectively (Fig. $2 d$ and Supplementary Fig. $2 \mathrm{c}, \mathrm{d})$. While this extension of both $\beta$ sheets by Zuol stabilizes the SBD, these differences also illustrate the plasticity of the SBD, which is underlined by the high $\mathrm{B}$ factors observed especially in the loop regions (Supplementary Fig. 3a,b). Notably, Zuo1N Phe23 inserts into a hydrophobic pocket between the upper and lower $\beta$-sheet of the Ssz1-SBD (Supplementary Fig. 3c,d). While this phenylalanine is not strictly conserved, the position is always occupied by a hydrophobic residue (e.g. Sc Val13) pointing to a structural role. In summary, the RAC core structure shows that ZuolN embraces the Ssz1$\mathrm{SBD} \beta$, resulting in an extended RAC-SBD.

A proline-rich motif in Zuo1N binds to the RAC-SBD. A third insight gained from the Ssz1-Zuo1N structure was that a conserved region (SLPLPTL, termed LP-motif, Fig. 2a) present in $C t Z u o 1 N$ between $\beta 0$ and $\beta 1$ binds to the RAC-SBD in a substrate-like manner (Fig. 2c-e). Canonical Hsp70s preferentially bind peptides containing hydrophobic residues at the central positions ${ }^{23-25}$. A detailed comparison of the RAC core with the DnaK-SBD structure with bound NRLLLTG peptide (NR-peptide, that serves as an Hsp70 model substrate) ${ }^{23}$ shows the conservation of the $\operatorname{SBD} \beta$ structures and their substrate binding modes (Fig. 2e, Supplementary Fig. 4a-d). The Zuol LPmotif and the NR-peptide overall establish similar interactions with their cognate SBDs. Zuo1 Leu10 occupies the hydrophobic center of the Ssz1 substrate binding pocket and corresponds to the central Leu ( 0 position) in the NRLLLTG peptide ${ }^{23}$. Residues Leu8, Pro9, Leu10 and Thr12 of the LP-motif establish H-bonds with backbone atoms of Ssz1-SBD $\beta$ strands $\beta 3, \beta 1, \beta 3$ and $\beta 4$, respectively, while the conserved Pro11 does not form a hydrogen bond (Supplementary Fig. 4b). The loop connecting $\beta 3$ and $\beta 4$ ( $\mathrm{L}_{\beta 3-\beta 4}$, disordered in the previous structure) is ordered due to stabilization by the LP-motif (Fig. 2e, Supplementary Fig. 4b,d). Interestingly, the position of the NR-peptide in $\mathrm{DnaK}^{23,26}$ and other Hsp70s, like BiP and human cytosolic Hsp7027,28, can be shifted by one residue due to crystal packing, which locates the third Leu to the central position (NRLLLTG), but maintains the hydrogen-bonding pattern. Notably, the Ssz1-bound LP-motif adopts an almost ideal poly-L-proline type II (PPII) helix, which resembles the binding mode of proline-rich antimicrobial peptides $^{26}$, while the DnaK-bound NR-peptide adopts a more extended conformation (Fig. 2e, Supplementary Fig. 4e,f). Overall, the LP-motif forms a PPII helix and binds to the RAC-SBD in cis like a pseudo-substrate. 
a

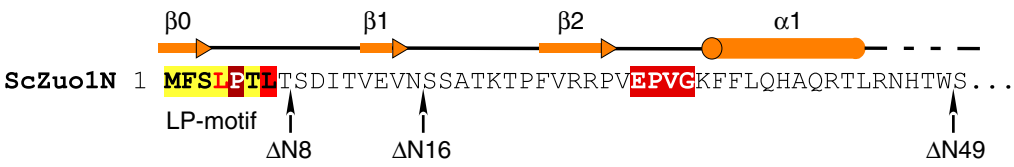

b
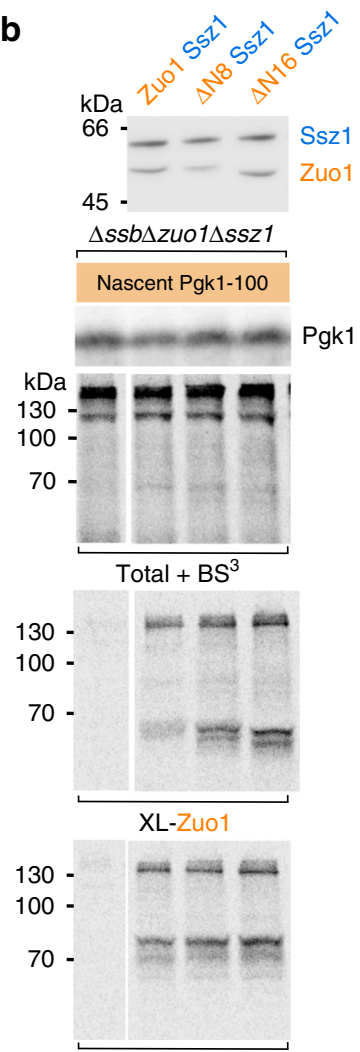

XL-Ssz1

e
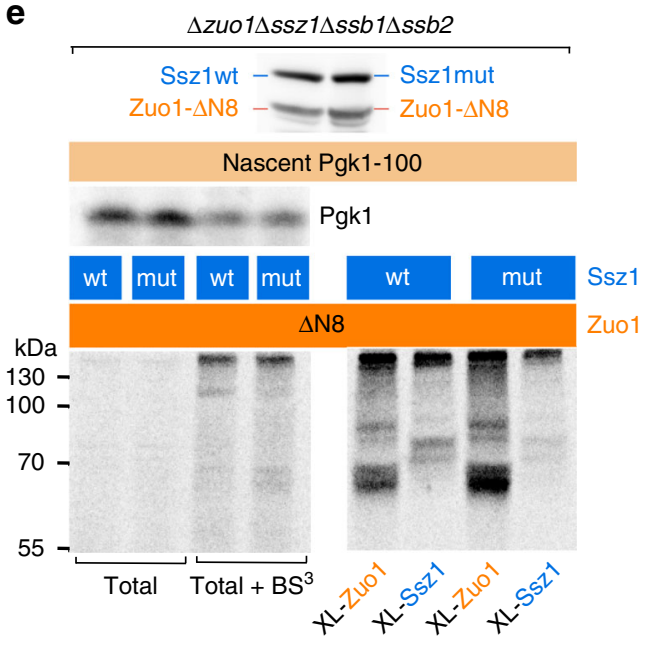

C
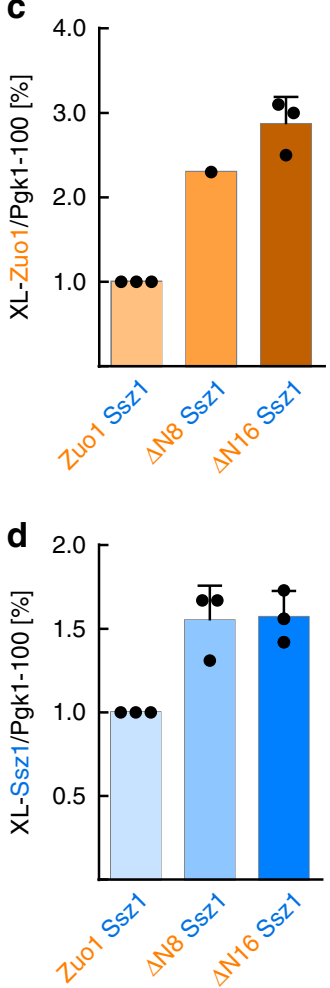

f

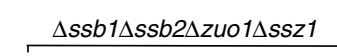
Zuo1-AN8 Ssz1

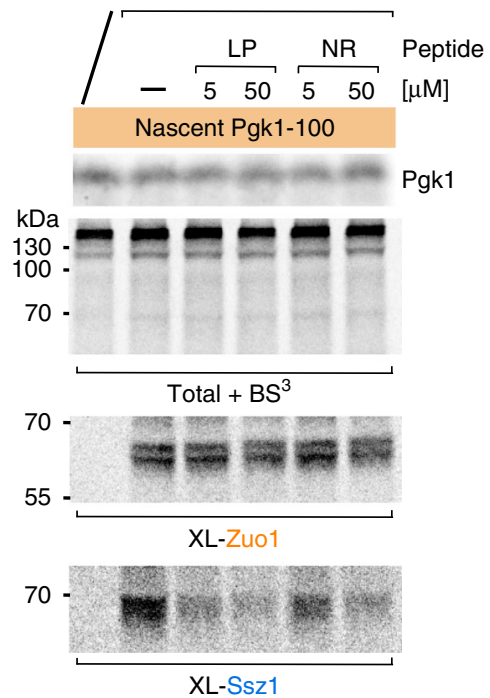

The RAC-SBD prefers the LP-motif over the NR-peptide. To analyze the RAC-SBD interaction with the LP-motif in more detail and to relate it to nascent chain binding in ScRAC (see below), we designed two $S c R A C$ variants comprising $S c S s z 1$ and either the $S c Z$ uo1N (residues 1-50, corresponding to $C t$ residues 1-60) or ScZuo1N $\Delta$ LP (residues 10-50) lacking the LP-motif.
Please note that while the LP-motif is conserved from fungi to human, the Zuo1N $\beta 0$ strand is shorter or absent in the human homolog MPP11 and in yeast (Fig. 2a). Nano differential scanning fluorimetry (nanoDSF) showed that deletion of the LP-motif drastically decreased the thermal stability of the RAC core complex from $52.9^{\circ} \mathrm{C}$ to $39.7^{\circ} \mathrm{C}$ (Supplementary Fig. 5a). Thus, 
Fig. 3 Nascent chain binding to the Ssz1-SBD is modulated by the $\mathbf{N}$-terminus of Zuo1. a Amino acid sequence of the ScZuo1N. Arrows indicate the positions of truncations in Zuo1- $\Delta$ N8, Zuo1- $\Delta$ N16, and Zuo1- $\Delta$ N49. The color code is as in Fig. 2a. The secondary structure derived from the crystal structure is depicted above. b The Zuo1N LP-motif interferes with the contact of nascent Pgk1-100 with Zuo1 and Ssz1. Ribosome-free extracts obtained from $\Delta s s b 1 \Delta s s b 2$ (Zuo1, Ssz1), $\Delta s s b 1 \Delta s s b 2$ RAC- $\Delta$ N8 ( $\Delta N$ 8, Ssz1), or $\Delta s s b 1 \Delta s s b 2$ RAC- $\Delta N 16$ ( $\Delta N 16$, Ssz1) strains (upper panel) were added to RNCs from $\Delta s s b 1 \Delta s s b 2 \Delta z u o 1 \Delta s s z 1$ translation extract prior to crosslinking. Crosslink products between Zuo1 and nascent Pgk1-100 (XL-Zuo1) and Ssz1 and nascent Pgk1-100 (XL-Ssz1), respectively. c, d Quantification of crosslinking between nascent Pgk1-100 and Zuo1 (c) and Ssz1 (d). Autoradiographs as shown in (b) were employed for the quantification of nascent Pgk1-100 and the crosslink products XL-Zuo1 and XL-Ssz1. Values were normalized as described in Methods. The efficiency of crosslinking is given as a percentage of nascent Pgk1-100 present in the reaction. Experiments were performed in triplicate, with the exception of the XL-Zuo1- $\Delta$ N8 (shown in $\mathbf{c}$ ), which was performed only once. Shown is the mean (bars) and the result of each biologically independent experiment (dots). Error bars indicate the standard deviation of the mean. e Crosslinking of the nascent chain to Ssz1 requires a functional peptide binding groove. Ribosome-free extracts from $\Delta s s b 1 \Delta s s b 2$ RAC- $\Delta$ N8 (Zuo1- $\Delta$ N8, Ssz1) or $\Delta s s b 1 \Delta s s b 2$ RACmut- $\Delta$ N8 (Zuo1- $\Delta$ N8, Ssz1mut) (upper panel) was added to RNCs generated in $\Delta s s b 1 \Delta s s b 2 \Delta z u 01 \Delta s s z 1$ translation extract as described in Fig. 1b. Crosslink products between Zuo1 and nascent Pgk1-100 (XLZuo1) and Ssz1 and nascent Pgk1-100 (XL-Ssz1), respectively. f The LP-peptide displaces the nascent chain from Ssz1. RNCs were generated in a $\Delta s s b 1 \Delta s s b 2 \Delta z$ uo1 1 ssz1 translation extract and ribosome-free extract from $\Delta s s b 1 \Delta s s b 2$ RAC- $\Delta N 8$ (Zuo1- $\Delta$ N8, Ssz1) was added. Subsequently, RNCs were isolated as described in Methods and LP-peptide (LP) or NR-peptide (NR) was added as indicated to the resuspended RNCs prior to the crosslinking reaction. Crosslink products between Zuo1 and nascent Pgk1-100 (XL-Zuo1) and Ssz1 and nascent Pgk1 (XL-Ssz1), respectively. Source data for (b-f) are provided as a Source Data file.

binding of the LP-motif to the RAC-SBD as a pseudo-substrate strongly stabilized the complex. We next asked whether free LPpeptide (MFSLPTL, corresponding to the LP-motif, ScZuol residues 1-7) was able to bind and thereby stabilize the complex. Indeed, addition of LP-peptide increased the melting temperature of Ssz1-Zuo1N $\Delta$ LP from 40.3 to $42.9^{\circ} \mathrm{C}$ (Supplementary Fig. 5b). This moderate, but significant effect on the thermal stability strongly suggested that the LP-peptide was able to bind to the Ssz1Zuo1N $\Delta$ LP complex in trans (see also below).

In order to quantify the interaction, we employed fluorescence anisotropy and compared the LP-peptide and the NR-peptide with respect to binding to the Ssz1-Zuo1N and Ssz1-Zuo1N $\Delta$ LP complex, respectively (Supplementary Fig. 6). While the affinity of the NR-peptide for both complexes was below the detection limit, the LP-peptide bound to the Ssz1-Zuo1N $\Delta$ LP complex with an estimated affinity of $11.9 \pm 4.0 \mu \mathrm{M}$, which is similar to the NRpeptide interacting with ATP-bound DnaK $(23.8 \pm 3.8 \mu \mathrm{M}$, lowaffinity $\left.\operatorname{state}^{29}\right)$. In order to confirm that the LP-peptide was indeed interacting with the peptide binding groove of Ssz1, a mutant was generated in which four point mutations (L439S/ K440P/I448F/G495K; Ssz1mut) were introduced into the peptide binding groove (Supplementary Fig. 5c). These mutations were based on previously characterized DnaK mutants deficient in substrate binding ${ }^{30}$. Analysis by nanoDSF showed that, in contrast to Ssz1-Zuo1N $\Delta \mathrm{LP}$, the melting temperature of Ssz1mut-Zuo1N $\Delta$ LP was basically not changed upon addition of the LP-peptide (Supplementary Fig. 5d). These data show that the LP-motif forming a PPII helix binds to the Ssz1-SBD as a lowaffinity pseudo-substrate, and suggest that this motif is optimized in sequence, structure, and positioning for binding to the RAC-SBD in cis. We hypothesize that the strictly conserved Pro11 (Pro5 in S. cerevisiae) plays a central role in restricting the conformation of the LP-motif for optimal binding. Therefore, the RAC-SBD seems to prefer the LP-motif over the Hsp70 model substrate NR-peptide.

The Zuo1 LP-motif modulates nascent chain binding to Ssz1. As the LP-motif occupies the RAC-SBD in cis and competes with peptide binding in trans (Supplementary Fig. 6), we speculated that the LP-motif might compete also with nascent chain binding to ribosome-bound RAC. To test this directly, we generated yeast strains expressing Zuol- $\Delta \mathrm{N} 8$, which carried a deletion of the LPmotif, or Zuo1- $\Delta$ N16, which lacked the LP-motif and Zuo1N $\beta 1$ (Fig. 3a). Zuo1- $\Delta$ N8 and Zuo1- $\Delta$ N16 formed stable complexes with Ssz1 (termed RAC- $\Delta \mathrm{N} 8$ and RAC- $\Delta \mathrm{N} 16$ ) (Supplementary Fig. 1e) and complemented growth defects of a $\Delta z u o 1$ strain when expressed in vivo (Supplementary Fig. 1i,j). The interaction of RAC- $\Delta$ N8 and RAC- $\Delta$ N16 with nascent Pgk1-100 was tested in the absence of Ssb, i.e. under conditions, in which wild type Zuol and Sszl efficiently interact with nascent chains (Fig. 1d and Supplementary Fig. 1b). Crosslinking of the nascent chain to both RAC subunits was enhanced in RAC- $\Delta \mathrm{N} 8$ or RAC- $\Delta \mathrm{N} 16$ complexes (Fig. $3 \mathrm{~b}-\mathrm{d}$ ). To test if the nascent chain was indeed bound to the peptide binding groove of Ssz1 when Zuol lacked the LP motif, we compared the crosslinking efficiency of the nascent chain to RAC- $\Delta \mathrm{N} 8$ (Zuol- $\Delta \mathrm{N} 8 / \mathrm{Ssz} 1$ ) with the crosslinking efficiency to RACmut- $\Delta$ N8 (Zuo1- $\Delta$ N8/Ssz1mut) (Fig. 3e). Indeed, the contact between the nascent chain and Sszlmut (Supplementary Fig. 5c,d) was strongly reduced when compared to Ssz1 (Fig. 3e). At the same time crosslinking of the nascent chain to Zuol- $\Delta$ N8 was enhanced (Fig. 3e). These observations are consistent with a model that the nascent chain is transferred from Zuol to the Ssz1-SBD. We next tested if free LP- or NR-peptide, added before the crosslinking reaction, affected nascent chain interaction with the RAC subunits. Neither of the peptides added prior to the crosslinking reaction affected nascent chain crosslinking to Zuo1- $\Delta \mathrm{N} 8$ (Fig. 3f, XL-Zuo1). However, addition of LP-peptide, and to a lesser extent also NR-peptide, significantly reduced crosslinking of the nascent chain to Ssz1 (Fig. 3f, XLSsz1). Thus, the LP-peptide was not only able to bind to the Ssz1Zuo1N $\Delta$ LP complex in trans (Supplementary Fig. 6), but also displaced a nascent chain from Ssz1 in the RAC- $\Delta \mathrm{N} 8$ complex, indicating that both substrates competed for the same binding site (Fig. 3f). Based on the combined structural and biochemical data we conclude that enhanced proximity of the nascent chain to Zuol- $\Delta$ N8/Zuol- $\Delta$ N16 was likely due to structural and/or positioning effects. In contrast, enhanced proximity of the nascent chain to Ssz1 reflected bona fide nascent chain binding to Ssz1$\operatorname{SBD} \beta$ resembling the low-affinity interaction of ATP-bound canonical Hsp70s with their substrates.

\section{Discussion}

In this work, we uncover mechanistically important features of the ribosome-bound $\mathrm{RAC} / \mathrm{Ssb}$ chaperone system. Combining crosslinking experiments with high resolution structure determination and substrate binding assays, we answer the longstanding question whether the role of Ssz1 is to structurally support the function of the J-domain protein Zuo1, or whether it acts as a chaperone. We show that Ssz1 indeed has chaperone activity, that Ssz1-SBD $\beta$ interacts with nascent chains and that this interaction is modulated by a conserved motif in ZuolN (LPmotif), which binds to the Ssz1-SBD like substrates bind to typical 

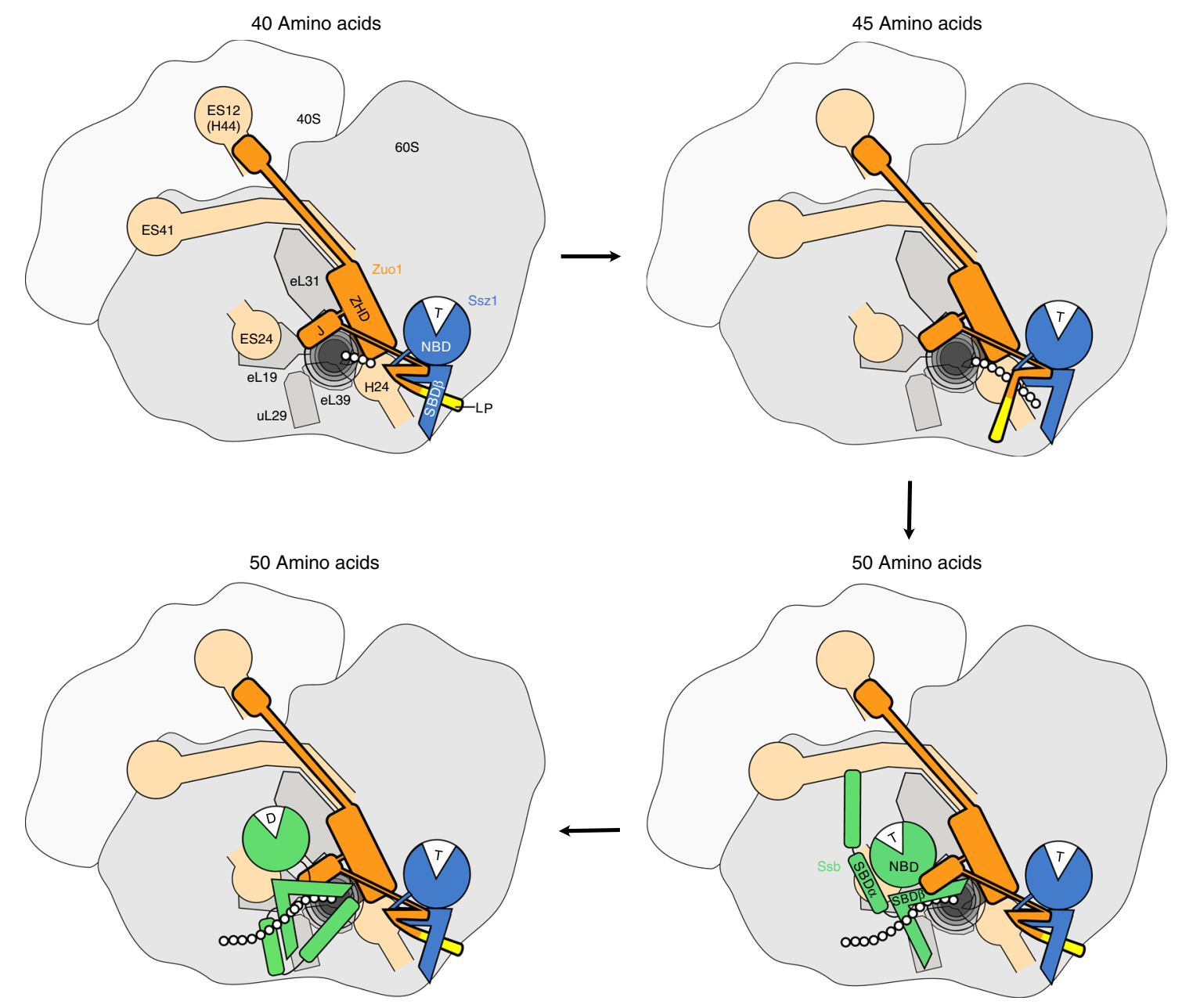

Fig. 4 Nascent chain handover at the ribosomal tunnel exit. Model of the RAC/Ssb substrate relay system at the ribosomal tunnel exit. At a length of 40 residues the nascent chain contacts Zuo1 (upper left panel). At a length of 45 residues the nascent chain reaches Ssz1 (upper right panel). This requires the displacement of the Zuo1 LP-motif from the Ssz1-SBD $\beta$. At 50 residues length, the nascent chain is transferred to Ssb, in a process that requires a direct transfer from the Ssz1-SBD $\beta$ to Ssb-SBD $\beta$, and a contact of the Zuo1 J-domain with the linker region of Ssb (lower right panel). The latter stimulates ATP hydrolysis by Ssb and traps the nascent chain in a Ssb.ADP.nascent chain complex (lower left panel). Likely in this situation the LP-motif interacts with the Ssz1-SBD $\beta$. The ribosomal $60 S$ and $40 S$ subunits are shown in dark and light gray. The tunnel exit is indicated in black, and residues of nascent Pgk1 are represented as white circles. Zuo1 (orange), Ssz1 (blue), Ssb (green), and Zuo1 LP-motif (yellow). J-domain (J), Zuotin homology domain (ZHD), nucleotide binding domain (NBD), substrate binding domain (SBD). Main contacts of RAC and Ssb with ribosomal proteins (gray) and RNA (sand) are indicated (for details on the ribosomal contacts of RAC and Ssb see ${ }^{4}$ ). For more details compare Discussion.

Hsp70s. To demonstrate that the growing nascent chain is transferred from Ssz1 to Ssb, the use of nascent chains shorter than 50 residues (Fig. 4) was fundamental. Longer nascent chains were efficiently captured by Ssb and for this reason were no longer in contact with RAC.

Ssz1 is commonly regarded a non-canonical Hsp70, because it lacks the SBDa lid domain ${ }^{4,17}$ and the Ssz1-NBD can bind, but cannot hydrolyze ATP 9,14 . The structure of the RAC core presented in this work reveals a novel property of Ssz1: Ssz1-SBD $\beta$ interacts with the Zuo1 LP-motif, which serves as a low-affinity inbuilt substrate. The consequences of these Ssz1-specific deviations from the general Hsp70 scheme can be understood by recalling that compared to wild type DnaK, a DnaK $\Delta$ lid variant is destabilized and shows reduced substrate affinity and an 10-100times faster $\mathrm{k}_{\mathrm{on}}$ and $\mathrm{k}_{\mathrm{off}}{ }^{31,32}$. Similarly, when the Hsp70 homolog $\mathrm{BiP}$ is AMPylated, ATP hydrolysis is inhibited and BiP is trapped in a low-substrate affinity ATP-like state ${ }^{33}$. In these cases, absence of the lid or inhibition of lid closure induces a destabilization of the SBD resulting in low-affinity substrate binding. Destabilization of Ssz1, induced by the loss of the lid domain, seems to be compensated by Zuo1N in two ways: by additional $\beta$-strands of Zuol that embrace the Ssz1-SBD, and by positioning of the LPmotif within the substrate binding site of the Ssz1-SBD. These stabilizing effects, which Zuo1 imposes on Ssz1, at the same time contribute to the trapping of Ssz1 in the low-substrate affinity, ATP-bound state. Our findings reveal that Ssz1 displays chaperone activity, which is seemingly optimized for the highly transient interactions required to cope with the situation at the ribosomal tunnel exit, where nascent chain substrates emerge at a rate of about 3-6 residues per second ${ }^{34,35}$. The specific properties of Ssz1 allow for a rapid forward transfer to Ssb within the RAC/Ssb chaperone triad. In line with this notion the Zuol LP-motif negatively modulates nascent chain binding to Ssz1. This previously unknown function of Zuol in some respect resembles a recently discovered supplementary function of the yeast Hsp70 nucleotide exchange factor (NEF) Fes $1^{36}$. Fes 1 does not only act as a NEF, but via its flexible $\mathrm{N}$-terminal domain mimics Hsp70 substrates. Accordingly, binding of the Fes1 N-terminal domain to the Hsp70-SBD promotes substrate release ${ }^{36}$. Ssz1 does not hydrolyze ATP and thus does not require a classical NEF 
as a co-chaperone. The above mentioned supplementary function of $\mathrm{NEFs}^{36}$, however, is seemingly adopted by the N-terminal LPmotif within the J-domain protein Zuol. In RAC, the intrinsic LP-motif is positioned close to the Ssz1-SBD, suggesting that permanent competition between the LP-motif and the emerging nascent chain safeguards the release of substrates and further supports forward transfer to Ssb. Interestingly, the LP-motif forms a PPII helix and its binding mode is very similar to prolinerich antimicrobial peptides that target DnaK (Supplementary Fig. $4 \mathrm{e}, \mathrm{f})^{26}$. Notably, the PPII helix is an abundant and versatile recognition motif with a restricted backbone conformation, which allows for sequence specific recognition without requiring particularly high affinity interaction.

Based on the data presented and previously published work on the interaction of RAC and Ssb with the ribosome $e^{4,10-12,22}$ we propose the following model for nascent chain handover at the ribosomal tunnel exit (Fig. 4).

At a length of 40 residues, an extended nascent chain predominantly contacts Zuol. Because nascent Pgk1 becomes accessible at a length of 36 residues $^{21}$, at that point, only four residues are exposed and only the $\alpha$-amino group of Met 1 is available for crosslinking. (Fig. 4, upper left panel). Therefore, Zuo1 residue(s), which crosslink to Pgk1-40, should be within a radius of about $25 \AA$ from the tunnel exit (considering that Met1 is about $15 \AA$ from the exit and the $\mathrm{BS}^{3}$ crosslinker bridges about $10 \AA)$. However, as Zuol does not contain one of the conserved domains, which enable a sub-class of J-domain proteins to interact with substrate proteins ${ }^{37}$, it is difficult to predict, which Zuo1 domain contacts the nascent chain. As Zuo1- $\Delta 49$ lacks the entire $\mathrm{N}$-terminal region, but still crosslinks to the nascent chain, Zuo1N is not, or at least not the only, contact of Zuol with the nascent chain. One candidate is the Zuol-ZHD, which directly contacts eL31 at the tunnel exit and is thus close to short nascent chains $^{12}$. The ZHD is confined to close Zuol homologs and may thus potentially constitute a novel type of substrate binding domain. This possibility will be addressed in future studies.

At a length of 45 residues, Pgk1 was mostly in contact with Ssz1 (Fig. 4, upper right panel). This contact was observed only when Ssz1 was bound to Zuol, indicating that Sszl had to be positioned at the tunnel exit to interact with a nascent chain. The unique properties of Ssz1 described in this study suggest that during translation, Ssz1-SBD $\beta$ does bind to the growing nascent chain, although not tightly, by that allowing for the continuous forward movement until the nascent chain gains contact with Ssb. As outlined above, Ssz1 seems optimized for transient low-affinity binding by specific deviations from the canonical Hsp70 mechanism. At a length of 50 residues, Pgk1 is mainly in contact with Ssb (Fig. 4, lower panels) ${ }^{5-7}$. Our data reveal that nascent Pgk1 was efficiently transferred from RAC to Ssb between a nascent chain length of 46-50 residues. Upon stimulation of the Ssb ATPase by the Zuo1 J-domain, Ssb switches to the high affinity conformation. If Ssb was absent, or was not bound to the ribosome, because it lacked its $\mathrm{C}$-terminal ribosome-binding region (Ssb- $\Delta \mathrm{C} 23)$, or if Sszl was absent or its substrate binding site blocked by mutagenesis, the growing nascent chain remained bound to RAC or Zuo1, respectively. These observations suggested that the Ssz1-SBD $\beta$ has to be in close proximity to the SsbSBD $\beta$ to allow for efficient direct nascent chain transfer.

Seemingly the RAC/Ssb system of yeast is optimized for efficient nascent chain transfer at the ribosome. However, yeast cells cope well with conditions, in which direct nascent chain transfer at the ribosomal tunnel to Ssb is much reduced, e.g. when cells express Ssb- $\Delta \mathrm{C} 23^{10,22}$. A possible explanation for this, at first glance, puzzling observation is provided by the finding that RAC remains in contact with the growing nascent chain when Ssb is not ribosome-bound. One should consider that RAC is unlikely fixed permanently to a single ribosome, but cycles between ribosomes and the cytosol ${ }^{4,5}$. Thus, transfer of nascent chains from RAC to Ssb may not only occur directly at the tunnel exit, but also in the cytosol. Such transfer may, however, also directly go to Ssa. It is tempting to speculate that in mammalian cells a similar mechanism of de novo protein folding has evolved. Mammalian cells do not possess an Hsp70 homolog, which interacts with ribosomes directly ${ }^{38}$. However, mammalian cells possess a RAC homolog, termed mRAC, which, just like its yeast counterpart, is fully ribosome-associated in cell extract ${ }^{39}$. Nevertheless mRAC is the cochaperone of the cytosolic Hsp70 homolog HSPA1A/B $4,38,39$. Of note, when heterologously expressed in yeast, mRAC complements growth defects of $\Delta z u o 1 \Delta s s z 1$ cells. Complementation by mRAC is independent of Ssb, as mRAC, even though ribosome-associated, cooperates with the yeast cytosolic Hsp70 homolog Ssa ${ }^{38}$. Taken together our data provide a starting point for detailed analyses of nascent chain interaction with the RAC/Ssb triad and its homologs at the ribosome to finally derive mechanistic insights into their function in protein homeostasis.

\section{Methods}

Strains, plasmids, and growth conditions. Strains and plasmids are listed in Supplementary Tables 1 and 2. All plasmids for expression of SSZ1 or ZUO1 variants in yeast contained $300 \mathrm{bp}$ up- and down-stream of the respective open reading frame. The pRS315-Zuol- $\Delta \mathrm{N} 8$ was obtained by QuikChange Lightning site-directed mutagenesis (Agilent Technologies) employing pRS315-Zuol ${ }^{17}$ as a template. pRS423-Zuol- $\Delta$ N8 was generated by transfer of Zuo1- $\Delta$ N8 from pRS315-Zuol- $\triangle \mathrm{N} 8$ to pRS423. The CtZUO1 sequence encoding for residues 1-60 was PCR-amplified from pET16b-His ${ }_{6}$-MBP-GSGSGS-TEV-CtZuo $1^{17}$ and was coligated with a PCR product of $\mathrm{His}_{6}-\mathrm{MBP}$ with a $3 \mathrm{C}$ sequence overhang, which encodes a 3C (Human Rhinovirus 3C precision protease) cleavage site (LEVLFQ/ $\mathrm{GP}$ ), into a pET16b vector using the XbaI and BamHI sites resulting in pET16bHis $_{6}$-MBP-3C-CtZuolN. The Saccharomyces cerevisiae sequence encoding for residues $1-50$ of Zuol was amplified by PCR from pRS315-Zuol ${ }^{17}$ and was also coligated with the $\mathrm{His}_{6}-\mathrm{MBP}-3 \mathrm{C}$ insert resulting in pET16b-His $-\mathrm{HBP}-3 \mathrm{C}-\mathrm{ScZuolN}$. pET16b-His ${ }_{6}$-MBP-3C-ScZuo1N $\Delta$ LP (residues 10-50) was obtained by QuikChange Lightning site-directed mutagenesis (Agilent Technologies). The coding sequence of $S c S S Z 1$ was amplified by PCR using $S c$ genomic DNA as a template and was cloned into pET24d-His ${ }_{6}$-SUMO or pRS315, resulting in pET24d-His $6_{6}^{-}$ SUMO-ScSsz1 or pRS315-ScSsz1. The L439S/K440P/I448F/G495K mutations were introduced into pYCPlac33-ScSsz $1^{19}$ by site-directed mutagenesis (QuikChange Lightning site-directed mutagenesis (Agilent Technologies)), resulting in pYCPlac33-Ssz1mut. ScSsz1 L439S/K440P/I448F/G495K was subsequently subcloned into pET24d-His ${ }_{6}$-SUMO resulting in pET24d-His ${ }_{6}$-SUMO-ScSsz1L439S/ K440P/I448F/G495K (pET24d-His 6 -SUMO-ScSsz1mut). Primers employed for cloning are shown in Supplementary Table 3.

The parental Saccharomyces cerevisiae wild type strain used in this study was $\mathrm{MH} 272-3 \mathrm{fa} / \mathrm{a}$ (ura3, leu2, his3, trp1, ade2) ${ }^{40}$. In the $\Delta s s z 1$ background, Zuol was expressed from a $2 \mu$ plasmid to obtain a Zuol expression level similar to the wild type $^{8}$. Yeast strains were grown in YPD complete medium (1\% yeast extract, $2 \%$ peptone, $2 \%$ glucose), or in SD minimal medium $(6.7 \mathrm{~g} / \mathrm{l}$ yeast nitrogen base without amino acids, $2 \%$ glucose) supplemented with the appropriate amino acids and nucleobases. Cultures were grown in liquid medium at $30^{\circ} \mathrm{C}$ with constant shaking at $200 \mathrm{rpm}$.

Preparation of purified Zuo1 complexes. pET16b-His ${ }_{6}$-MBP-3C-CtZuo1N was coexpressed with pET24d-His ${ }_{6}$-SUMO-strep-CtSsz1 and purified as described ${ }^{17}$ with the following modifications. Dialysis was performed in $20 \mathrm{mM}$ Hepes/KOH $\mathrm{pH} 7.5,50 \mathrm{mM} \mathrm{NaCl}, 10 \mathrm{mM} \mathrm{KCl}, 5 \mathrm{mM} \mathrm{MgCl}_{2}$ and $1 \mathrm{mM}$ DTT containing 3C protease. The sample was then applied to a $1 \mathrm{ml}$ ResourceQ column (GE Healthcare) and eluted with a $0-1 \mathrm{M} \mathrm{NaCl}$ gradient. The size-exclusion chromatography was performed in $20 \mathrm{mM}$ Hepes/KOH pH 7.5, $150 \mathrm{mM} \mathrm{NaCl}, 10 \mathrm{mM} \mathrm{KCl}, 5 \mathrm{mM}$ $\mathrm{MgCl}_{2}$ and $1 \mathrm{mM}$ DTT.

pET16b-His ${ }_{6}$-MBP-3C-ScZuo1N or pET16b-His - $_{6}$ MBP-3C-ScZuo1N $\Delta$ LP was coexpressed with pET24d-His ${ }_{6}-\mathrm{SUMO}-\mathrm{ScSsz1}$ or pET24d-His ${ }_{6}-\mathrm{SUMO}-\mathrm{ScSsz1}$ mut and purified as above with the following modified buffer composition. Dialysis buffer was $20 \mathrm{mM}$ Hepes/KOH pH 7.5, $50 \mathrm{mM} \mathrm{KOAc,} 5 \mathrm{mM} \mathrm{Mg}(\mathrm{OAc})_{2}$ and $1 \mathrm{mM}$ DTT. The ResourceQ elution was performed with a $0-1 \mathrm{M}$ KOAc gradient. The size-exclusion buffer was $20 \mathrm{mM}$ Hepes/KOH pH 7.5, $120 \mathrm{mM}$ KOAc, $5 \mathrm{mM} \mathrm{Mg}$ $(\mathrm{OAc})_{2}$ and $1 \mathrm{mM}$ DTT.

ScSsb1 was expressed from pET24d-His ${ }_{6}$-SUMO-Ssb1 and purified as above with the following modification. The nickel affinity chromatography column (IMAC) was washed with buffer containing $50 \mathrm{mM} \mathrm{NaCl}$ and $5 \mathrm{mM}$ ATP and subsequently with buffer containing $1 \mathrm{M} \mathrm{NaCl}$ and $5 \mathrm{mM}$ ATP. The eluate was 
dialyzed against $20 \mathrm{mM}$ Hepes/KOH pH 7.5, $120 \mathrm{mM} \mathrm{KOAc,} 5 \mathrm{mM} \mathrm{Mg}(\mathrm{OAc})_{2}$ and $1 \mathrm{mM}$ DTT in the presence of Ulpl protease. The cleaved SUMO tag was removed by reverse IMAC, the Ssb1-containing flow-through was applied onto a ResourceQ and then eluted with a 0-1 M KOAc gradient. Ssb1-containing fractions were dialyzed against $20 \mathrm{mM}$ Hepes/ $\mathrm{KOH} \mathrm{pH} \mathrm{7.5,} 120 \mathrm{mM} \mathrm{KOAc}, 5 \mathrm{mM} \mathrm{Mg}(\mathrm{OAc})_{2}$ and $1 \mathrm{mM}$ DTT, shock frozen in liquid nitrogen and stored at $-80^{\circ} \mathrm{C}$.

Crystallization and structure determination. Crystallization screens were performed at $291 \mathrm{~K}$ by the sitting-drop vapor-diffusion method upon mixing equal volumes $(0.2 \mu \mathrm{l})$ of the $C t$ Ssz1-Zuo1N protein solution $(10 \mathrm{mg} / \mathrm{ml})$ and reservoir solution containing 20.5\% (v/v) PEG 3350 and $0.2 \mathrm{M}$ ammonium acetate. Crystals grew after $21 \mathrm{~h}$ and were fished after 5 days. The crystals were cryo-protected by transfer into cryo-solution containing mother liquor and 20\% (v/v) glycerol, and flash-frozen in liquid nitrogen. Diffraction data were measured under cryogenic conditions (100 K; Oxford Cryosystems Cryostream) at the European Synchrotron Radiation Facility (ESRF, Grenoble) beamline id30a $3^{41}$.

Data were processed with $\mathrm{XDS}^{42}$. Phases were obtained by molecular replacement using PHASER; ${ }^{43}$ search model $5 \mathrm{MB} 9^{17}$ in the CCP4I2 software package ${ }^{44,45}$. Iterative model building and refinement were done with COOT $^{46}$ and phenix.refine $e^{47}$. The validation was performed using EDSTATS ${ }^{48}$ and MOLPROBITY $^{49}$ included in CCP4I2. UCSF chimera ${ }^{50}$ was used for figure preparation, always using chains $\mathrm{C}$ and $\mathrm{D}$. Sequence alignments were performed using Clustal Omega ${ }^{51}$ and visualized with ESPript 3.0;52 http://espript.ibcp.fr/ ESPript/ESPript/.

Ribosome-binding assays. Yeast strains were grown to early log phase on YPD, prior to harvest cycloheximide was added to a final concentration of $100 \mu \mathrm{g} / \mathrm{ml}$, and subsequently cells were collected via centrifugation at $5000 \mathrm{~g}$. Cell pellets were resuspended in ribosome-binding buffer $(20 \mathrm{mM}$ Hepes/KOH pH 7.4, $2 \mathrm{mM} \mathrm{Mg}$ $(\mathrm{OAc})_{2}, 120 \mathrm{mM} \mathrm{KOAc}, 100 \mu \mathrm{g} / \mathrm{ml}$ cycloheximide, $2 \mathrm{mM}$ DTT, $1 \mathrm{mM}$ PMSF, protease inhibitor mix) and total cell extracts were prepared by the glass beads method ${ }^{53}$. After a clearing spin at $20.000 \mathrm{~g}$, each $60 \mu \mathrm{l}$ of the total glass beads extract $\left(\mathrm{A}_{260}\right.$ between 80 and $\left.100 \mathrm{mAU}\right)$ was loaded onto a $90 \mu \mathrm{l}$ sucrose cushion (25\% sucrose, $20 \mathrm{mM}$ Hepes/KOH pH 7.4, $120 \mathrm{mM} \mathrm{KOAc,} 2 \mathrm{mM} \mathrm{Mg}(\mathrm{OAc})_{2}$, $2 \mathrm{mM}$ DTT, $1 \mathrm{mM}$ PMSF, protease inhibitor mix). After centrifugation at $400.000 \mathrm{~g}$ at $4{ }^{\circ} \mathrm{C}$ for $25 \mathrm{~min}$ the cytosolic supernatant was collected and the ribosomal pellet was resuspended in $300 \mu \mathrm{l}$ ribosome-binding buffer. Aliquots of the total cell extract, cytosolic supernatant, and resuspended ribosomal pellets were precipitated by addition of TCA (trichloroacetic acid) to a final concentration of 5\%. TCA pellets were dissolved in SDS-sample buffer and were analyzed on 10\% Tris-Tricine gels followed by immunoblotting.

Preparation of yeast translation extract. Yeast translation extract was prepared from 10 to 121 cultures $\left(\mathrm{OD}_{600} 0.8-1.0\right)$ of yeast strains as indicated in Results and Figure Legends. The protocol followed the method previously described by Walter and coworkers ${ }^{54}$. Collected cells were washed once with water and were then resuspended in $200 \mathrm{ml}$ sorbitol buffer (1.4 M sorbitol, $50 \mathrm{mM}$ K-phosphate buffer pH 7.4, $10 \mathrm{mM}$ DTT) containing $2 \mathrm{mg}$ zymolyase $20 \mathrm{~T}$ (nacalai tesque Inc.)/g cells. After incubation at $30^{\circ} \mathrm{C}$ with agitation at $120 \mathrm{rpm}$ for $30 \mathrm{~min}$, spheroblasts were collected at $4{ }^{\circ} \mathrm{C}$ and were resuspended in $300 \mathrm{ml}$ sorbitol buffer (YPD medium containing $1 \mathrm{M}$ sorbitol). After incubation at $22^{\circ} \mathrm{C} / 120 \mathrm{rpm}$ for $90 \mathrm{~min}$, spheroblasts were re-collected, and were resuspended in $400 \mathrm{ml}$ sorbitol buffer. The spheroblast suspension was transferred into two $500 \mathrm{ml} \mathrm{JA}-10$ (Beckman Coulter) centrifuge tubes and was underlaid with $200 \mathrm{ml}$ cold sorbitol buffer. After centrifugation at $4.000 \mathrm{~g}$ for $7 \mathrm{~min}$ at $4{ }^{\circ} \mathrm{C}$ in a JA-10 rotor, spheroblasts were washed twice with cold sorbitol buffer and were then resuspended in 5-10 $\mathrm{ml}$ lysis buffer (20 mM Hepes/KOH pH 7.4, $100 \mathrm{mM}$ KOAc, $2 \mathrm{mM} \mathrm{MgOAc}_{2}, 2 \mathrm{mM}$ DTT, $0.5 \mathrm{mM}$ PMSF, $1 \times$ protease inhibitor mix). The spheroblast suspension was transferred into a $40 \mathrm{ml}$ dounce homogenizer (Kontes Glass $\mathrm{Co}$.) and spheroblasts were disrupted by douncing with a type B pestle on ice. The resulting extract was centrifuged in a SS34 rotor (Piramoon Technologies Inc.) at $15.000 \mathrm{rpm}$ for $18 \mathrm{~min}$ at $4{ }^{\circ} \mathrm{C}$. The supernatant was collected and was centrifuged in a $70.1 \mathrm{Ti}$ rotor (Beckman Coulter) at $38.000 \mathrm{rpm}$ for $35 \mathrm{~min}$ at $4{ }^{\circ} \mathrm{C}$. The supernatant was loaded onto a Superdex-G25 gel filtration column equilibrated with gel filtration buffer $(20 \mathrm{mM}$ Hepes/KOH pH 7.4, $100 \mathrm{mM} \mathrm{KOAc,} 2$ mM MgOAc 2,2 mM DTT, 0.5 mM PMSF, $20 \%$ glycerol) at a flow rate of $1.5 \mathrm{ml} / \mathrm{min}$. Peak fractions with the highest $\mathrm{A}_{260}$ were pooled, were supplemented with $1 \mathrm{mM} \mathrm{CaCl}$, and were treated with $300 \mathrm{U} / \mathrm{ml}$ micrococcal nuclease (Roche) for $15 \mathrm{~min}$ at $20^{\circ} \mathrm{C}$. Nuclease treatment was terminated by the addition of $2 \mathrm{mM}$ EGTA and aliquots of the extract, termed yeast translation extract, were frozen in liquid nitrogen and were stored at $-80^{\circ} \mathrm{C}$.

In vitro transcription and translation. DNA templates for transcription reactions were generated by PCR using pSPUTK-Pgk ${ }^{20}$ as a template. Reverse primers were designed such, that PCR products encoded different length PGK1 sequences, which all lacked a stop codon. The length of the resulting translation products, which remain ribosome-bound, is indicated in Results and Figure Legends. Transcripts were generated using SP6 polymerase (Thermo Fischer Scientific) as previously described ${ }^{55}$. RNCs were generated via translation reactions primed with the stop codon-less transcripts at $20^{\circ} \mathrm{C}$ in the presence of $\left[{ }^{35} \mathrm{~S}\right]$-methionine
(Hartmann-Analytic) for $80 \mathrm{~min}^{20,54}$. Translation reactions were stopped by the addition of $50 \mu \mathrm{g} / \mathrm{ml}$ cycloheximide final concentration and subsequently RNCs were isolated via centrifugation at $400.000 \mathrm{~g}$ for $20 \mathrm{~min}$ in a TLA-100 or TLA-100.2 rotor (Beckman Coulter) ${ }^{20}$.

Chemical crosslinking and immunoprecipitation. Chemical crosslinking of ribosome-bound nascent chains to Ssb, Zuol, or Ssz1 was analyzed essentially as described ${ }^{20}$. In brief, resuspended RNCs were incubated in the presence of $400 \mu \mathrm{M}$ BS $^{3}$ (bis-(sulfosuccinimidyl)-suberate, spacer length $1.14 \mathrm{~nm}$, Thermo Scientific) for 20 min on ice and crosslinking was then quenched by the addition of TRIS base (tris(hydroxymethyl)aminomethane) to a final concentration of $50 \mathrm{mM}$. Crosslinked protein samples were precipitated by the addition of TCA (final concentration 5\%), pellets were collected by centrifugation and were dissolved in dissociation buffer (200 mM TRIS/HCl pH 7.5, 4\% SDS; 10 mM EDTA, $100 \mu \mathrm{g} / \mathrm{ml}$ BSA, protease inhibitor mix, $1 \mathrm{mM}$ PMSF). Totals shown in Figs. 1,3 and Supplementary Fig. 1 before (total) and after $\mathrm{BS}^{3}$ crosslinking (total $+\mathrm{BS}^{3}$ ) represent $5 \%$ of the dissolved material employed for immunoprecipitation reactions. The resulting denatured protein samples were used to identify crosslink products between Ssb, Zuol, or Ssz1 and nascent chains via affinity purification under denaturing conditions. To that end, protein A sepharose beads (GE Healthcare) were pre-coated with antibodies directed against Ssb, Zuol, or Sszl and subsequently the denatured crosslinked material was allowed to bind to the beads resuspended in immunoprecipitation buffer (10 mM TRIS/HCl pH 7.5, $150 \mathrm{mM}$ $\mathrm{NaCl}, 5 \mathrm{mM}$ EDTA, $1 \%$ Triton X-100, protease inhibitor mix, $0.5 \mathrm{mM}$ PMSF) ${ }^{20}$. Crosslink products bound to protein A sepharose beads were released by addition of SDS-sample buffer at $95^{\circ} \mathrm{C}$ for $10 \mathrm{~min}$. Samples were analyzed on Tris-Tricine gels ${ }^{56}$ followed by autoradiography. Loading was normalized according to the signal of $\left[{ }^{35} \mathrm{~S}\right]$-labelled nascent Pgk1 in the total prior to crosslinking. Low exposure autoradiographs of nascent chains in the totals (labelled Pgk1) are shown in Fig. 1 and Fig. 3. Relative crosslinking efficiencies between nascent Pgk1-100 and Zuol or Ssz1, respectively were determined as follows. For each biological replicate, band intensities of non-crosslinked nascent Pgk1-100 and of Pgk1-100 crosslink products (XL-Zuo1 or XL-Ssz1) was determined on a single autoradiograph for wild type RAC and the different mutant variants of RAC. The crosslinking efficiency (XL-Zuo1/Pgk1-100 or XL-Ssz1/Pgk1-100) of each sample was calculated and subsequently the ratio of XL-Zuo1/Pgk1-100 or XL-Ssz1/Pgk1-100 in the presence of wild type RAC was set to 1 in order to normalize the biological replicates (see Fig. 3c,d). The original data are provided as a Source Data file.

Preparation of ribosome-free extracts. For complementation experiments translation reactions were performed in either $\Delta s s b 1 \Delta s s b 2$ or $\Delta s s b 1 \Delta s s b 2 \Delta z u o 1 \Delta s s z 1$ translation extract as indicated. Complementation with purified Ssb1 was performed by adding $\mathrm{His}_{6}$-tagged $S c \mathrm{Ssb} 1$ at a final concentration of $2 \mu \mathrm{M}$ to the translation reaction prior to the addition of mRNA. Ribosome-free cytosolic extract of various mutant yeast strains for complementation experiments as indicated in Results and Figure Legends was generated as follows. The $\mathrm{A}_{260}$ of a total cell extract prepared by the glass beads method (see above) was determined, and the total cell extract was subsequently adjusted to a final concentration of $800 \mathrm{mM} \mathrm{KOAc.} \mathrm{Under} \mathrm{this} \mathrm{con-}$ dition ribosome-bound proteins such as Ssb, Zuol and Ssz1 are released from ribosomes. Ribosomes were then collected by ultracentrifugation at $400.000 \mathrm{~g}$ for $25 \mathrm{~min}$ at $4{ }^{\circ} \mathrm{C}$ and the resulting supernatant (ribosome-free extract) was re-adjusted to a concentration of $120 \mathrm{mM}$ KOAc by addition of KOAc-free resuspension buffer (20 mM Hepes/KOH pH 7.4, $2 \mathrm{mM} \mathrm{Mg}(\mathrm{OAc})_{2}, 50 \mu \mathrm{g} / \mathrm{ml}$ cycloheximide, $1 \mathrm{mM}$ PMSF, protease inhibitor mix). Ribosome-free extract was added to Ssb/RAC-free RNCs after completion of translation reactions. The amount of ribosome-free extract added to RNCs was adjusted such that the total $\mathrm{A}_{260}$ of the added ribosomefree extract equaled the $\mathrm{A}_{260}$ of the yeast translation reaction. After incubation for $5 \mathrm{~min}$ at $20^{\circ} \mathrm{C}$, RNCs and bound factors were isolated via sedimentation at $400.000 \mathrm{~g}$ and were resuspended in $300 \mu \mathrm{l}$ resuspension buffer prior to crosslinking analysis, which was performed as described above.

Binding affinity measurements using fluorescence anisotropy. The protein buffers were exchanged for anisotropy buffer $(20 \mathrm{mM}$ Hepes/KOH pH 7.5, $200 \mathrm{mM}$ $\mathrm{KCl}, 5 \mathrm{mM} \mathrm{MgCl} 2,1 \mathrm{mM} \mathrm{DTT}$ ) using Zeba spin columns (Thermo Scientific). $1 \mu \mathrm{M}$ BSA was added to prevent unspecific binding of the fluorescently labelled peptide to the microplate (Greiner Bio-One opaque 384 well plate). Fluorescence anisotropy measurements were performed using the LP-peptide (MFSLPTL) or the NR-peptide (NRLLLTG) labelled at the $\mathrm{N}$ terminus with fluorescein (Peptide Specialty Laboratories GmbH, Heidelberg). Two-fold serial dilutions of each protein (ScSsz1-Zuo1N or ScSsz1-Zuo1N $\Delta$ LP) were mixed in a 1:1 ratio with $40 \mathrm{nM}$ peptides in anisotropy buffer in 384-well opaque plates (Greiner Bio One) and were incubated at room temperature for $30 \mathrm{~min}$. Measurements were performed in triplicate using a plate reader (SpectraMax M5e Multi Mode Microplate reader, Molecular Devices). To determine the binding constant $\left(K_{\mathrm{D}}\right)$ the data were fitted to a one-site binding equation using Python ${ }^{57}$.

NanoDSF measurements. The melting temperature of ScSsz1-Zuo1N, ScSsz1Zuo1N $\triangle$ LP or ScSszlmut-Zuo1NALP with or without the LP-peptide (Peptide Specialty Laboratories GmbH, Heidelberg) were determined using the Prometheus 
NT.48 nanoDSF system using nano-DSF grade capillaries (NanoTemper Technologies $\mathrm{GmbH}$ ). The measurements were performed in $20 \mathrm{mM}$ Hepes/KOH pH 7.5, $200 \mathrm{mM} \mathrm{KCl}, 5 \mathrm{mM} \mathrm{MgCl}_{2}, 1 \mathrm{mM}$ DTT. The LP-peptide, which was dissolved in DMSO, was pre-diluted in the above buffer such that the final DMSO concentration in the experiment was below $0.5 \%$. DMSO diluted in the same way was added to the negative control. Intrinsic protein fluorescence was measured continuously at $330 \mathrm{~nm}$ and $350 \mathrm{~nm}$ with a temperature gradient ranging from 20 to $90^{\circ} \mathrm{C}$ at a rate of $1.5^{\circ} \mathrm{C} / \mathrm{min}$.

Statistics and Reproducibility. Figures 1, 3, and Supplementary Fig. 1 include representative autoradiographs and immunoblots. The experiments were performed at least for the following number of times: Fig. 1c, two times; Fig. 1d, two times; Fig. 1e, three times; Fig. 1f, three times; Fig. 3b-d, see Figure Legend and Source Data file; Fig. 3e, two times; Fig. $3 \mathrm{f}$ two times; Supplementary Fig. 1a, two times; Supplementary Fig. 1b, two times; Supplementary Fig. 1c, three times; Supplementary Fig. 1d, one time; Supplementary Fig. 1e, two times; Supplementary Fig. 1f, two times; Supplementary Fig. 1g, two times; Supplementary Fig. 1h, three times; Supplementary Fig. 1i, two times; Supplementary Fig. 1j, two times; Supplementary Fig. 6, three times.

Miscellaneous. Proteins were separated on $10 \%$ Tris-Tricine gels ${ }^{56}$. Antibodies for immunoblotting and denaturing immunoprecipitation reactions are rabbit polyclonal antibodies (Rospert lab antibody collection). Antibody dilutions were as follows: $\alpha$-Ssb 1:5.000, $\alpha$-Ssz1 1:20.000, $\alpha$-Zuo1 1:40.000, $\alpha$-Sse1 1:20.000, $\alpha$-Rps9 1:8.000, $\alpha$-Rpl4 1:20.000, and $\alpha$-Pgk1 1:20.000. Horseradish-conjugated goat antirabbit IgG (Pierce catalog number 31460, 1:10.000) was employed as a secondary antibody. Immunoblots were developed by enhanced chemiluminescence ${ }^{58}$. Protease inhibitor mix ( $1 \times$ ) contained $1.25 \mu \mathrm{g} / \mathrm{ml}$ leupeptin, $0.75 \mu \mathrm{g} / \mathrm{ml}$ antipain, 0.25 $\mu \mathrm{g} / \mathrm{ml}$ chymostatin, $0.25 \mu \mathrm{g} / \mathrm{ml}$ elastinal, and $5 \mu \mathrm{g} / \mathrm{ml}$ pepstatin A. Quantification of digital phosphorimages (autoradiographs) was performed using AIDA ImageAnalyzer (Raytest). At least 3 independent biological replicates were performed for statistical analysis, which was conducted with GraphPad Prism (version 6.07).

Reporting summary. Further information on experimental design is available in the Nature Research Reporting Summary linked to this paper.

\section{Data availability}

Accession codes: Coordinates and structure factors have been deposited in the Protein Data Bank under accession code 6SR6. The PDB datasets 5MB9, 1DKZ and 4EZP have been used in this study. The source data underlying Figs. 1c-f, 3b-f, and Supplementary Figs. 1a-j, 5a-c, and 6a,b are provided as a Source Data file. Other data that support the findings of this study are available from the corresponding authors on reasonable request.

Received: 17 September 2019; Accepted: 3 March 2020;

Published online: 20 March 2020

\section{References}

1. Preissler, S. \& Deuerling, E. Ribosome-associated chaperones as key players in proteostasis. Trends Biochem. Sci. 37, 274-283 (2012).

2. Kramer, G., Shiber, A. \& Bukau, B. Mechanisms of Cotranslational Maturation of Newly Synthesized Proteins. Annu. Rev. Biochem. 88, 337-364 (2019).

3. Peisker, K., Chiabudini, M. \& Rospert, S. The ribosome-bound Hsp70 homolog Ssb of Saccharomyces cerevisiae. Biochim. Biophys. Acta 1803, 662-672 (2010).

4. Zhang, Y., Sinning, I. \& Rospert, S. Two chaperones locked in an embrace: Structure and function of the ribosome-associated complex RAC. Nat. Struct. Mol. Biol. 24, 611-619 (2017).

5. Hundley, H. et al. The in vivo function of the ribosome-associated Hsp70, Ssz1, does not require its putative peptide-binding domain. Proc. Natl Acad. Sci. USA 99, 4203-4208 (2002).

6. Gautschi, M. et al. The yeast $\mathrm{N}^{\alpha}$-acetyltransferase NatA is quantitatively anchored to the ribosome and interacts with nascent polypeptides. Mol. Cell. Biol. 23, 7403-7414 (2003).

7. Döring, K. et al. Profiling Ssb-Nascent Chain Interactions Reveals Principles of Hsp70-Assisted Folding. Cell 170, 298-311 e220 (2017).

8. Gautschi, M., Mun, A., Ross, S. \& Rospert, S. A functional chaperone triad on the yeast ribosome. Proc. Natl Acad. Sci. USA 99, 4209-4214 (2002).

9. Huang, P., Gautschi, M., Walter, W., Rospert, S. \& Craig, E. A. The Hsp70 Ssz1 modulates the function of the ribosome-associated J-protein Zuol. Nat. Struct. Mol. Biol. 12, 497-504 (2005).

10. Gumiero, A. et al. Interaction of the cotranslational Hsp70 Ssb with ribosomal proteins and rRNA depends on its lid domain. Nat. Commun. 7, 1-12 (2016).

11. Zhang, Y. et al. Structural basis for interaction of a cotranslational chaperone with the eukaryotic ribosome. Nat. Struct. Mol. Biol. 21, 1042-1046 (2014).
12. Lee, K., Sharma, R., Shrestha, O. K., Bingman, C. A. \& Craig, E. A. Dual interaction of the Hsp70 J-protein cochaperone Zuotin with the 40S and 60S ribosomal subunits. Nat. Struct. Mol. Biol. 23, 1003-1010 (2016).

13. Peisker, K. et al. Ribosome-associated complex binds to ribosomes in close proximity of Rpl31 at the exit of the polypeptide tunnel in yeast. Mol. Biol. Cell 19, 5279-5288 (2008).

14. Leidig, C. et al. Structural characterization of a eukaryotic chaperone-the ribosome-associated complex. Nat. Struct. Mol. Biol. 20, 23-28 (2013).

15. Ducett, J. K. et al. Unfolding of the C-terminal domain of the J-protein Zuol releases autoinhibition and activates Pdr1-dependent transcription. J. Mol. Biol., 425, 19-31 (2013).

16. Mayer, M. P. Hsp70 chaperone dynamics and molecular mechanism. Trends Biochem. Sci. 38, 507-514 (2013).

17. Weyer, F. A., Gumiero, A., Gese, G. V., Lapouge, K. \& Sinning, I. Structural insights into a unique Hsp70-Hsp40 interaction in the eukaryotic ribosomeassociated complex. Nat. Struct. Mol. Biol. 24, 144-151 (2017)

18. Mayer, M. P. \& Kityk, R. Insights into the molecular mechanism of allostery in Hsp70s. Front. Mol. Biosci. 2, 58 (2015).

19. Conz, C. et al. Functional characterization of the atypical Hsp70 subunit of yeast ribosome-associated complex. J. Biol. Chem. 282, 33977-33984 (2007).

20. Raue, U., Oellerer, S. \& Rospert, S. Association of protein biogenesis factors at the yeast ribosomal tunnel exit is affected by the translational status and nascent polypeptide sequence. J. Biol. Chem. 282, 7809-7816 (2007).

21. Zhang, Y., Wölfle, T. \& Rospert, S. Interaction of nascent chains with the ribosomal tunnel proteins Rpl4, Rpl17, and Rpl39 of Saccharomyces cerevisiae. J. Biol. Chem. 288, 33697-33707 (2013).

22. Hanebuth, M. A. et al. Multivalent contacts of the Hsp70 Ssb contribute to its architecture on ribosomes and nascent chain interaction. Nat. Commun. 7 , 13695 (2016)

23. Zhu, X. et al. Structural analysis of substrate binding by the molecular chaperone DnaK. Science 272, 1606-1614 (1996).

24. Bertelsen, E. B., Chang, L., Gestwicki, J. E. \& Zuiderweg, E. R. Solution conformation of wild-type E. coli Hsp70 (DnaK) chaperone complexed with ADP and substrate. Proc. Natl Acad. Sci. USA 106, 8471-8476 (2009).

25. Gragerov, A., Zeng, L., Zhao, X., Burkholder, W. \& Gottesman, M. E. Specificity of DnaK-peptide binding. J. Mol. Biol. 235, 848-854 (1994).

26. Zahn, M. et al. Structural studies on the forward and reverse binding modes of peptides to the chaperone DnaK. J. Mol. Biol. 425, 2463-2479 (2013).

27. Zhang, P., Leu, J. I., Murphy, M. E., George, D. L. \& Marmorstein, R. Crystal structure of the stress-inducible human heat shock protein 70 substratebinding domain in complex with peptide substrate. PLOS ONE 9, e103518 (2014).

28. Yang, J., Nune, M., Zong, Y., Zhou, L. \& Liu, Q. Close and allosteric opening of the polypeptide-binding site in a Human Hsp70 Chaperone BiP. Structure 23, 2191-2203 (2015).

29. Gisler, S. M., Pierpaoli, E. V. \& Christen, P. Catapult mechanism renders the chaperone action of Hsp70 unidirectional. J. Mol. Biol. 279, 833-840 (1998)

30. Montgomery, D. L., Morimoto, R. I. \& Gierasch, L. M. Mutations in the substrate binding domain of the Escherichia coli $70 \mathrm{kDa}$ molecular chaperone, DnaK, which alter substrate affinity or interdomain coupling. J. Mol. Biol. 286, 915-932 (1999).

31. Buczynski, G., Slepenkov, S. V., Sehorn, M. G. \& Witt, S. N. Characterization of a lidless form of the molecular chaperone DnaK: deletion of the lid increases peptide on- and off-rate constants. J. Biol. Chem. 276, 27231-27236 (2001).

32. Moro, F., Fernandez-Saiz, V. \& Muga, A. The lid subdomain of DnaK is required for the stabilization of the substrate-binding site. J. Biol. Chem. 279, 19600-19606 (2004)

33. Preissler, S. \& Ron, D. Early events in the endoplasmic reticulum unfolded protein response. Cold Spring Harb. Perspect. Biol. 11, a033894 (2019).

34. Ingolia, N. T., Lareau, L. F. \& Weissman, J. S. Ribosome profiling of mouse embryonic stem cells reveals the complexity and dynamics of mammalian proteomes. Cell 147, 789-802 (2011).

35. Yan, X., Hoek, T. A., Vale, R. D. \& Tanenbaum, M. E. Dynamics of Translation of Single mRNA Molecules In Vivo. Cell 165, 976-989 (2016).

36. Gowda, N. K. C. et al. Nucleotide exchange factors Fes1 and HspBP1 mimic substrate to release misfolded proteins from Hsp70. Nat. Struct. Mol. Biol. 25, 83-89 (2018).

37. Kampinga, H. H. \& Craig, E. A. The HSP70 chaperone machinery: J proteins as drivers of functional specificity. Nat. Rev. Mol. Cell. Biol. 11, 579-592 (2010).

38. Jaiswal, H. et al. The chaperone network connected to human ribosomeassociated complex (mRAC). Mol. Cell. Biol. 31, 1160-1173 (2011).

39. Otto, H. et al. The chaperones MPP11 and Hsp70L1 form the mammalian ribosome-associated complex. Proc. Natl Acad. Sci. USA 102, 10064-10069 (2005).

40. Heitman, J., Movva, N. R., Hiestand, P. C. \& Hall, M. N. FK 506-binding protein proline rotamase is a target for the immunosuppressive agent FK 506 in Saccharomyces cerevisiae. Proc. Natl Acad. Sci. USA 88, 1948-1952 (1991). 
41. Theveneau, P. et al. The upgrade programme for the structural biology beamlines at the European Synchrotron Radiation Facility-high throughput sample evaluation and automation. J. Phys.: Conf. Ser. 425, 012001 (2013).

42. Kabsch, W. XDS. Acta Crystallogr. D: Biol. Crystallogr. 66, 125-132 (2010).

43. McCoy, A. J. et al. Phaser crystallographic software. J. Appl. Crystallogr. 40, 658-674 (2007).

44. Winn, M. D. et al. Overview of the CCP4 suite and current developments. Acta Crystallogr. D: Biol. Crystallogr. 67, 235-242 (2011).

45. Potterton, L. et al. CCP4i2: the new graphical user interface to the CCP4 program suite. Acta Crystallogr. D: Struct. Biol. 74, 68-84 (2018).

46. Emsley, P., Lohkamp, B., Scott, W. G. \& Cowtan, K. Features and development of Coot. Acta Crystallogr. D 66, 486-501 (2010).

47. Afonine, P. V. et al. Towards automated crystallographic structure refinement with phenix.refine. Acta Crystallogr. D: Biol. Crystallogr. 68, 352-367 (2012).

48. Tickle, I. J. Statistical quality indicators for electron-density maps. Acta Crystallogr. D: Biol. Crystallogr. 68, 454-467 (2012).

49. Chen, V. B. et al. MolProbity: all-atom structure validation for macromolecular crystallography. Acta Crystallogr. D. Biol. Crystallogr. 66, 12-21 (2010).

50. Pettersen, E. F. et al. UCSF Chimera-a visualization system for exploratory research and analysis. J. Comput. Chem. 25, 1605-1612 (2004).

51. Sievers, F. et al. Fast, scalable generation of high-quality protein multiple sequence alignments using Clustal Omega. Mol. Syst. Biol. 7, 539 (2011).

52. Robert, X. \& Gouet, P. Deciphering key features in protein structures with the new ENDscript server. Nucleic Acids Res. 42, W320-324 (2014).

53. Ashe, M. P., De Long, S. K. \& Sachs, A. B. Glucose depletion rapidly inhibits translation initiation in yeast. Mol. Biol. Cell 11, 833-848 (2000).

54. Garcia, P. D., Hansen, W. \& Walter, P. In vitro protein translocation across microsomal membranes of Saccharomyces cerevisiae. Methods Enzymol. 194, 675-682 (1991).

55. Fünfschilling, U. \& Rospert, S. Nascent polypeptide-associated complex stimulates protein import into yeast mitochondria. Mol. Biol. Cell. 10, 3289-3299 (1999).

56. Schägger, H. \& von Jagow, G. Tricine-sodium dodecyl sulfate-polyacrylamide gel electrophoresis for the separation of proteins in the range from 1 to 100 kDa. Anal. Biochem. 166, 368-379 (1987).

57. Rossum, G. Python Reference Manual (CWI (Centre for Mathematics and Computer Science), 1995).

58. Chiabudini, M. et al. Release factor eRF3 mediates premature translation termination on polylysine-stalled ribosomes in Saccharomyces cerevisiae. Mol. Cell. Biol. 34, 4062-4076 (2014).

\section{Acknowledgements}

The authors thank C. Siegmann from the BZH/Cluster of Excellence:CellNetworks crystallization platform for protein crystallization, and acknowledge access to the beamlines at the European Synchrotron Radiation Facility (ESRF) in Grenoble and the support of the beamline scientists. We acknowledge A. Hendricks, J.P. Kreysing, M. McDowell and D. Layer for technical assistance. We thank G. Stier for providing plasmids and K. Wild for valuable comments on the structure and the manuscript. This work was funded by the Deutsche Forschungsgemeinschaft (DFG) through the Leibniz programme (SI 586/6-1) and Project-ID 201348542 - SFB 1036, TP22 (to I.S.), Project-ID 403222702 - SFB 1381, TP B08 (to S.R.), and RO 1028/5-1 (to S.R.), and by the Excellence Initiative of the German federal and state governments [BIOSS-2] (to S.R) and [CellNetworks] (to I.S.). I.S. is an investigator of the Cluster of Excellence: CellNetworks.

\section{Author contributions}

Y.Z., G.V.G., C.C., K.L., S.R., and I.S. designed the experiments and analyzed the data. G. V.G. and J.K. solved the crystal structure. Y.Z., G.V.G., C.C., T.W., and K.L. performed experiments. Y.Z., G.V.G., K.L, S.R., and I.S. wrote the manuscript. All authors commented on the manuscript.

\section{Competing interests}

The authors declare no competing interests.

\section{Additional information}

Supplementary information is available for this paper at https://doi.org/10.1038/s41467 020-15313-w.

Correspondence and requests for materials should be addressed to S.R. or I.S

Peer review information Nature Communications thanks Yury Chernoff and the other, anonymous, reviewer(s) for their contribution to the peer review of this work. Peer reviewer reports are available.

Reprints and permission information is available at http://www.nature.com/reprints

Publisher's note Springer Nature remains neutral with regard to jurisdictional claims in published maps and institutional affiliations.

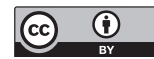

Open Access This article is licensed under a Creative Commons Attribution 4.0 International License, which permits use, sharing, adaptation, distribution and reproduction in any medium or format, as long as you give appropriate credit to the original author(s) and the source, provide a link to the Creative Commons license, and indicate if changes were made. The images or other third party material in this article are included in the article's Creative Commons license, unless indicated otherwise in a credit line to the material. If material is not included in the article's Creative Commons license and your intended use is not permitted by statutory regulation or exceeds the permitted use, you will need to obtain permission directly from the copyright holder. To view a copy of this license, visit http://creativecommons.org/ licenses/by/4.0/.

(c) The Author(s) 2020 